UNIVERSIDADE DE SÃO PAULO

FACULDADE DE MEDICINA DE RIBEIRÃO PRETO

\title{
LICÉRIO MIGUEL
}

Cirurgia para o câncer de endométrio: comparação das abordagens laparotômica e laparoscópica 


\section{LICÉRIO MIGUEL}

Cirurgia para o câncer de endométrio: comparação das abordagens laparotômica e laparoscópica

Dissertação apresentada à Faculdade de Medicina de Ribeirão Preto da Universidade de São Paulo, como requisito para a obtenção do Título de Mestre em Ciências Médicas.

Área de Concentração: Ginecologia e Obstetrícia.

Orientador: Prof. Dr. Francisco José Candido dos Reis

Ribeirão Preto

2019 
Autorizo a reprodução e divulgação total ou parcial deste trabalho, por qualquer meio convencional ou eletrônico, para fins de estudo e pesquisa, desde que citada a fonte.

Miguel, Licério

Cirurgia para o câncer de endométrio: comparação das abordagens laparotômica e laparoscópica / Licério Miguel. Orientador: Prof. Dr. Francisco José Candido dos Reis. - 2019. 60 f.

Dissertação (Mestrado) - Programa de Pós-Graduação em Ginecologia e Obstetrícia, Faculdade de Medicina de Ribeirão Preto, Universidade de São Paulo, Ribeirão Preto, 2019.

Orientador: Reis, Francisco José Candido dos

Versão original

1. Câncer de endométrio 2. Cirurgia 3. Laparotomia 4. Laparoscopia 


\section{AGRADECIMENTOS}

Agradeço aos meus saudosos pais Arnaldo e Maria do Carmo, que, com tanta dedicação, viveram e construíram a nossa família. Agradeço aos meus irmãos e minha irmã pela oportunidade da convivência, compartilhando das nossas diferenças e alegrias. Agradeço às minhas cunhadas, cunhados e sobrinhos e aos meus sogros pela parceria e compreensão de sempre.

À minha esposa Marcela, compreensiva, amorosa e fiel companheira, que tanto agradeço e admiro, permitindo-me construir um alicerce familiar, consolidado com a chegada de nossa filha Helena. Tenho grande orgulho em poder seguir nesta jornada com vocês!

Agradeço ao meu orientador Professor Doutor Francisco José Candido dos Reis, pela disponibilidade e paciência, tornando este mestrado uma forma ímpar de enxergar o mundo acadêmico com novos olhos, proporcionando ainda mais conhecimento e crescimento.

Agradeço a todos os professores e mestres com os quais tive contato e contribuíram para a minha formação pessoal e profissional, desde minha infância.

Agradeço ao Departamento de Ginecologia e Obstetrícia da Faculdade de Medicina de Ribeirão Preto da Universidade de São Paulo o qual me proporcionou a oportunidade da residência médica e da pós-graduação, com agradecimento especial a todos os seus funcionários.

Agradeço à Faculdade de Medicina de Botucatu da Universidade Estadual Paulista “Júlio de Mesquita Filho", responsável pela minha graduação, dando início a uma nova etapa em minha vida, repleta de muito estudo mas também de muita realização profissional.

Agradeço ao SAM - Serviço de Arquivo Médico do Hospital das Clínicas da Faculdade de Medicina de Ribeirão Preto da Universidade de São Paulo, que viabilizou a análise dos prontuários de forma extremamente efetiva e solícita.

E, por último mas com relevância primária, a todas as pacientes que, através de suas enfermidades, me permitiram aprender e produzir este trabalho. Nossa dedicação será válida para que melhorias sempre ocorram na assistência a vocês, com a garantia do melhor tratamento e qualidade de vida. 
MIGUEL, L. Cirurgia para o câncer de endométrio: comparação das abordagens laparotômica e laparoscópica. 2019. 60 f. Dissertação (Mestrado) - Faculdade de Medicina de Ribeirão Preto, Universidade de São Paulo, 2019.

Aprovado em:

Banca Examinadora

Prof. Dr.

Instituição:

Julgamento:

Prof. Dr.

Instituição:

Julgamento:

Prof. Dr.

Instituição:

Julgamento:

Prof. Dr.

Instituição:

Julgamento:

Prof. Dr.

Instituição:

Julgamento: 


\section{RESUMO}

MIGUEL, L. Cirurgia para o câncer de endométrio: comparação das abordagens laparotômica e laparoscópica. 2019. 60 f. Dissertação (Mestrado) - Faculdade de Medicina de Ribeirão Preto, Universidade de São Paulo, 2019.

Introdução: $O$ câncer de endométrio é a segunda neoplasia ginecológica mais frequente no Brasil. A cirurgia é o procedimento primário na maioria dos casos e tem como objetivo fundamental o estadiamento da doença, sendo na maioria das vezes também o tratamento definitivo. É característica das pacientes com esta doença a presença de obesidade e de comorbidades associadas. O uso de técnicas minimamente invasivas para a sua abordagem tem sido cada vez mais empregado e discutido. Objetivo: Comparar os aspectos referentes à abordagem cirúrgica por via aberta ou laparoscópica para o câncer endometrial, avaliando as duas possibilidades, suas complicações, recorrência e sobrevida. Métodos: Foram levantados os dados de todas as pacientes submetidas ao tratamento cirúrgico para o câncer endometrial a partir de janeiro de 1998, quando se iniciou a abordagem laparoscópica no serviço de Oncologia Ginecológica do Hospital das Clínicas da Faculdade de Medicina da Universidade de São Paulo, Ribeirão Preto (HC FMRP-USP), até dezembro de 2017. Durante esse período, a cirurgia minimamente invasiva e a cirurgia aberta tradicional foram usadas no serviço, de acordo com a preferência do cirurgião, como forma de abordagem dessas pacientes. A análise foi baseada em dados dos registros médicos do paciente: tipo de cirurgia, resultados a curto prazo, resultados a longo prazo e fatores intercorrentes. Resultados: Do total de 400 mulheres incluídas neste estudo, 316 foram submetidas à laparotomia e 84 à laparoscopia. As mulheres submetidas à laparotomia apresentaram maior frequência de tumores grau 3 (22,8\% vs. 11,9\%; $\mathrm{p}=0,006)$ e maior frequência de quimioterapia adjuvante $(14,6 \%$ vs. $6 \% ; \mathrm{p}=0,055)$. A laparoscopia foi associada ao aumento do tempo cirúrgico (194,7 min vs. 165,1 min; $\mathrm{p}<0,001)$ e redução da taxa de complicações cirúrgicas (0 vs. 9,2\%; p =0,008). A laparoscopia não aumentou o risco de recorrência (HR: 0,43, IC 95\% 0,18-1,02; p=0,06) e aumentou a sobrevida global em dez anos (HR: 0,44, IC 95\% 0,22-0,91; p = 0,03). Conclusão: A laparoscopia apresentou vantagens claras sobre a laparotomia para o câncer endometrial precoce. Foi associado a uma menor taxa de complicações cirúrgicas e melhor sobrevida global.

Palavras-chave: Câncer de endométrio. Cirurgia. Laparotomia. Laparoscopia. 


\begin{abstract}
MIGUEL, L. Endometrial cancer surgery: comparison of laparotomic and laparoscopic approaches. 2019. 60 f. Dissertação (Mestrado) - Faculdade de Medicina de Ribeirão Preto, Universidade de São Paulo, Ribeirão Preto, 2019.

Introduction: Endometrial cancer is the second most common gynecological cancer in Brazil. Surgery is the primary procedure in most cases and its main objective is the staging of the disease, most of the time also being the definitive treatment. It is characteristic of the patients with this disease the presence of obesity and associated comorbidities. The use of minimally invasive techniques for its approach has been increasingly employed and discussed. Objective: To compare the aspects regarding the open or laparoscopic surgical approach for endometrial cancer, evaluating the two surgical possibilities and their complications, recurrence and survival. Methods: Data were collected from all patients undergoing surgical treatment for endometrial cancer from January 1998, when the laparoscopic approach was initiated at the Gynecological Oncology service of the Clinics Hospital of Ribeirão Preto Medical School until December 2017. During this period, minimally invasive surgery and traditional open surgery were used in our service, according to the surgeon's preference to approach these patients. The analysis was based on data from the patient's medical records: type of surgery, short-term outcome, long-term outcome, and intercurrent factors. Results: From a total of 400 women were included in this study, 316 undergone to laparotomy and 84 to laparoscopy. Women submitted to laparotomy presented higher frequency of grade 3 tumors $(22.8 \%$ vs. $11.9 \% ; \mathrm{p}=$ $0.006)$ and higher frequency of adjuvant chemotherapy ( $14.6 \%$ vs. $6 \% ; p=0.055)$. Laparoscopy was associated with increased surgical time (194.7 min vs. $165.1 \mathrm{~min} ; \mathrm{p}<0.001)$, and reduced rate of surgical complications ( 0 vs. $9.2 \% ; p=0.008$ ). Laparoscopy did not increase the risk of recurrence (HR: $0.43,95 \% \mathrm{CI} 0.18-1.02 ; \mathrm{p}=0.06)$ and increased the ten-years overall survival (HR: 0.44, 95\% CI 0.22-0.91; $\mathrm{p}=0.03$ ). Conclusion: Laparoscopy presented clear advantages over laparotomy for early endometrial cancer. It was associated with a lower rate of surgical complications and better overall survival.
\end{abstract}

Keywords: Endometrial cancer. Surgery. Laparotomy. Laparoscopy. 


\section{LISTA DE ILUSTRAÇÕES}

Figura 1 - Sobrevida global de acordo com o tipo de cirurgia ..................................... 42

Figura 2 - Comprovante de submissão do artigo ......................................................... 43

Figura 3 - Fluxograma da casuística do estudo ........................................................ 50 


\section{LISTA DE TABELAS}

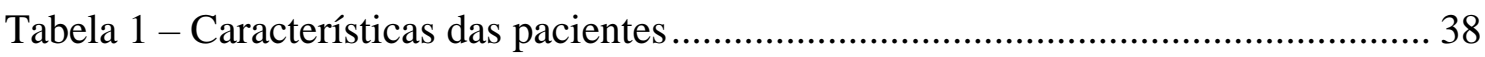

Tabela 2 - Resultados de acordo com o tipo de cirurgia ............................................. 39

Tabela 3 - Modelo de riscos proporcionais de Cox para mortalidade por todas as causas.

Tabela 4 - Modelo de riscos proporcionais de Cox para recorrência 


\section{LISTA DE SIGLAS}

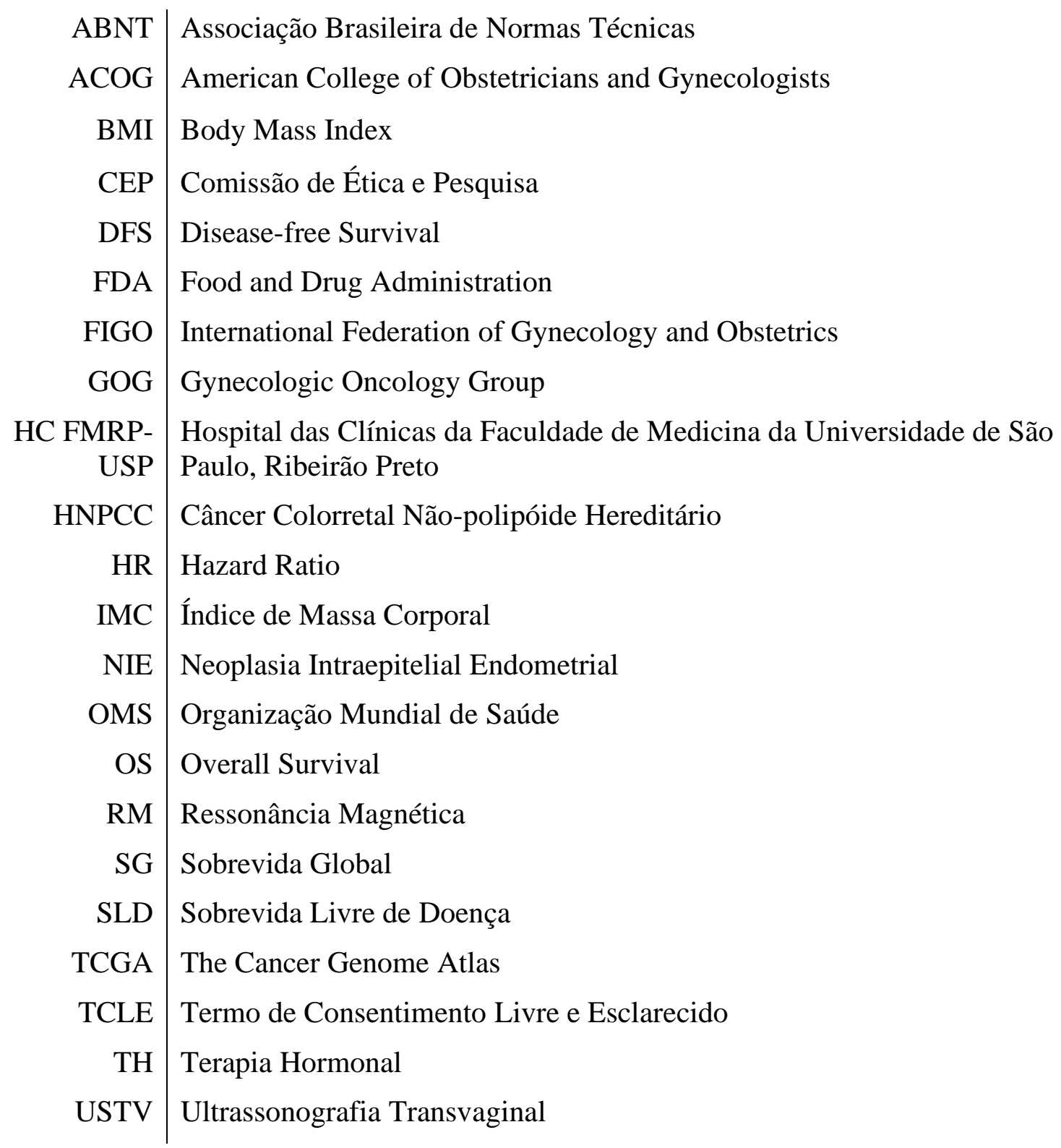




\section{Sumário}

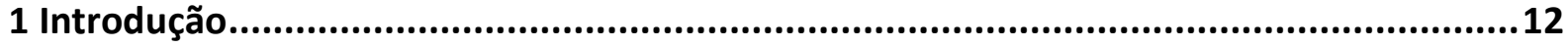

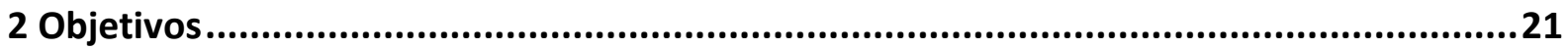

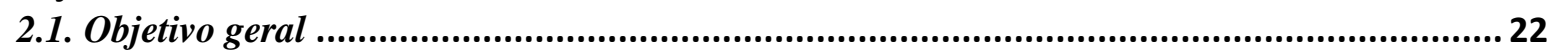

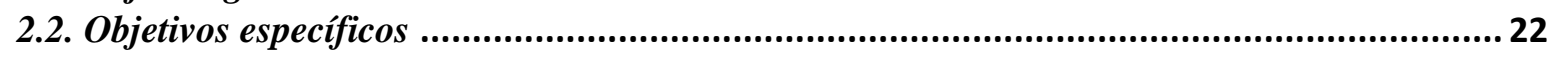

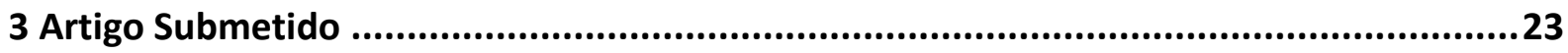

Title page

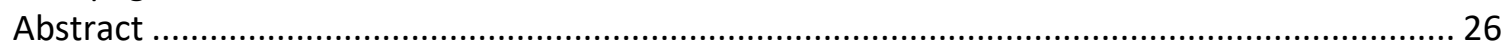

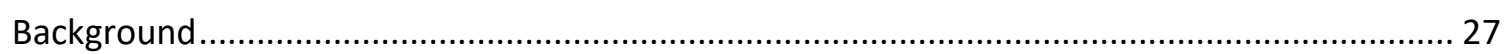

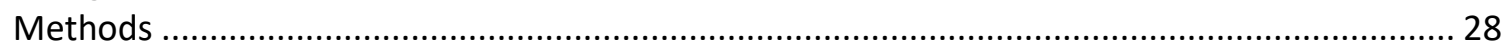

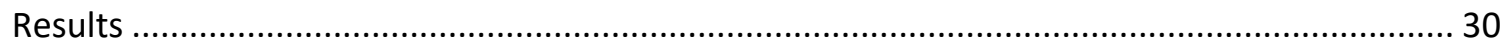

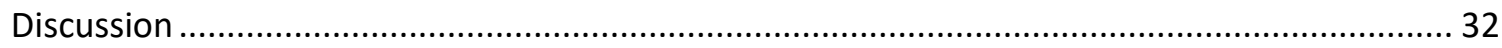

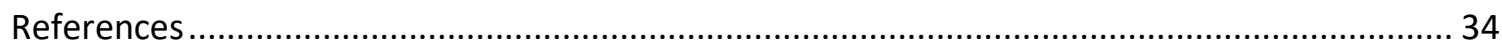

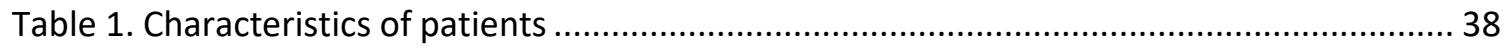

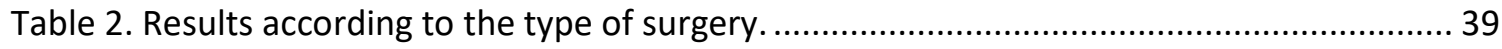

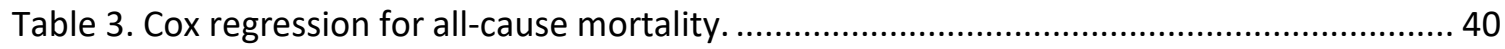

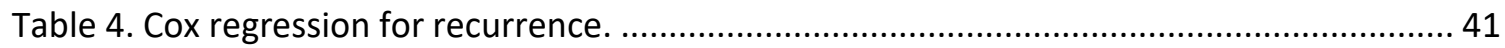

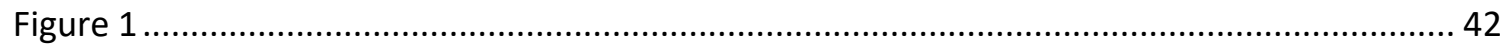

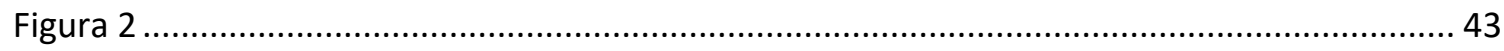

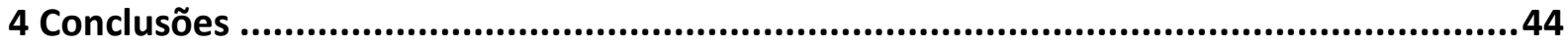

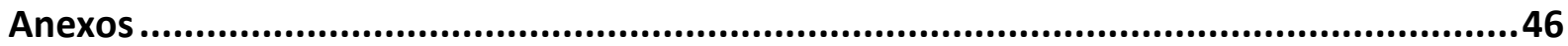

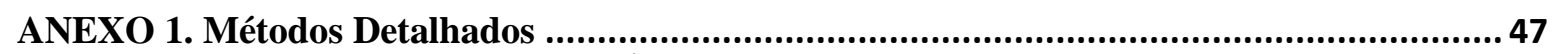

ANEXO 2. Aprovação do Comitê de Ética........................................................................ 51

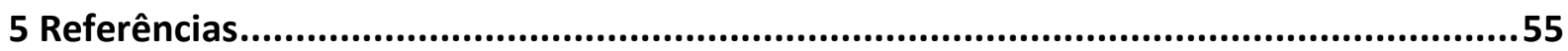


1 Introdução 
O câncer de endométrio é o mais comum entre as mulheres nos Estados Unidos, representando $6 \%$ de todos os tumores malignos no sexo feminino, com uma estimativa de 61880 novos casos e 12160 óbitos para o ano de 2019 (SIEGEL; MILLER; JEMAL, 2019). No Brasil, corresponde ao sexto mais frequente (COORDENAÇÃO, 2016), com uma estimativa de 6600 novos casos para cada ano do biênio 2018-2019 (SANTOS, 2018). As mulheres caucasianas têm um risco de 2,88\% ao longo da vida de desenvolvimento de câncer de corpo uterino em comparação com um risco de 1,69\% para mulheres africanas e/ou americanas (BURKE et al., 2014).

Comparado a outros tumores, o prognóstico das pacientes com câncer de endométrio é bom, com uma sobrevida em 10 anos de 77,6\% (Reino Unido) (PETO et al., 2000). Como o diagnóstico na maioria dos casos acontece em estádios iniciais, a cirurgia é curativa, assumindo papel crucial no tratamento desta patologia (ELTABBAKH et al., 2000).

Foram descritos diversos fatores de risco para o desenvolvimento do câncer de endométrio, e de modo geral, a maioria deles está associado, direta ou indiretamente, à exposição estrogênica excessiva (SÉNÉCHAL et al., 2015). Dentre esses fatores, a obesidade é a causa mais frequente de produção endógena em excesso deste hormônio, configurando o principal fator de risco para o desenvolvimento do câncer endometrial (BERGSTRÖM et al., 2001a). Nos últimos dez anos, observou-se um aumento da incidência dessa doença em mulheres mais jovens nos Estados Unidos, provavelmente em decorrência do aumento da obesidade de início precoce (MOORE; BREWER, 2017). A utilização da terapia hormonal (TH) com utilização de estrogênio exógeno sem oposição da progesterona é o segundo fator de risco mais importante, o que leva à prescrição rotineira da terapia combinada para mulheres na pós-menopausa que ainda tenham útero, como forma de prevenção (ALLEN et al., 2010). Os fatores menstruais e reprodutivos, associados à anovulação crônica ou exposição hormonal prolongada (menarca precoce, menopausa tardia ou nuliparidade), aumentam o risco de desenvolvimento da neoplasia (HARRIS; TERRY, 2016). Outro fator importante relaciona-se ao histórico familiar, representado principalmente pela Síndrome de Lynch, sendo o câncer de endométrio a manifestação extracolônica mais comum no câncer colorretal não-polipóide hereditário (HNPCC) (LYNCH et al., 2015), com risco de 40 a 60\% de desenvolvimento da doença em pacientes portadoras da mutação nos genes MLH1 e MSH2. Comorbidades como diabetes melito e hipertensão arterial também estão mais comumente associadas ao câncer de endométrio, bem como idade avançada (SOROSKY, 2012). O uso de tamoxifeno aumenta em 
duas a três vezes o risco de desenvolvimento da doença, em virtude do efeito proliferativo sobre o endométrio (CHLEBOWSKI et al., 2015).

A maioria dos cânceres de endométrio surge com a progressão de lesões hiperplásicas histologicamente identificáveis, classificadas de acordo com a Organização Mundial de Saúde (OMS). A nova classificação de 2014, aceita pela Sociedade Internacional de Patologistas Ginecológicos, dividiu a hiperplasia em dois grupos: hiperplasia benigna e hiperplasia atípica / neoplasia intraepitelial endometrial (NIE). O esquema da OMS 2014 é mais provável de identificar, com sucesso, lesões pré neoplásicas, em relação à antiga classificação de 1994 (SOBCZUK; SOBCZUK, 2017). Embora haja essa divisão didática, há uma heterogeneidade morfológica entre as pacientes, e somente algumas características preservadas são úteis como critérios diagnósticos. A grande maioria dos tumores de corpo uterino (97\% dos casos) correspondem aos carcinomas endometriais (ROSE, 1996).

O câncer endometrial é classificado em dois tipos patológicos com comportamentos biológicos diversos. O tipo I geralmente é relacionado à exposição ao estrogênio, ocorrendo em pacientes mais jovens, obesas ou na perimenopausa; geralmente são tumores de baixo grau e que surgem em um contexto de hiperplasia endometrial atípica, representando a maioria dos casos (75\%). O tipo histológico mais comum é o adenocarcinoma endometrióide. Os tumores do tipo II geralmente representam as neoplasias de alto grau, sendo os tipos histológicos mais comuns com células serosas ou claras, geralmente com invasão miometrial mais profunda, comportamento agressivo e não decorrentes de lesões precursoras, sendo mais comuns em mulheres negras e com idade mais avançada, representando aproximadamente $10 \%$ dos casos. Os demais casos são classificados em síndromes hereditárias, sendo a Síndrome de Lynch responsável pela metade desses quadros (SOROSKY, 2012).

Na década passada, a utilização de sequenciamentos genômicos levou à descoberta de aberrações patogênicas adicionais em cada um dos subtipos histológicos. Com os dados do atlas genômico de tumores - The Cancer Genome Atlas (TCGA) - e o avanço no entendimento da biologia molecular do câncer de endométrio, a doença foi reclassificada em quatro subgrupos distintos: subtipo POLE ultramutado, instabilidade de microssatélite (MSI), baixo número de cópias $(\mathrm{CNL})$ e alto número de cópias ( $\mathrm{CNH}$ - serous-like). Em relação ao comportamento tumoral, o primeiro grupo apresenta o melhor e o último o pior prognóstico (BELL; ELLENSON, 2018).

Atualmente não é preconizada nenhuma estratégia de rastreamento específica para câncer endometrial, exceto para casos de HNPCC, com recomendação de início de triagem 
entre as idades de 30 a 35 anos (exame físico detalhado, educação sobre sintomas anormais, biópsia endometrial anual em pacientes na pré-menopausa, realização de ultrassonografia transvaginal e dosagem de CA-125) (CRISPENS, 2012).

O sintoma mais comum associado ao diagnóstico de câncer de endométrio é o sangramento vaginal anormal (BURKE et al., 2014), devendo-se ter especial atenção para pacientes na pré-menopausa, considerando que múltiplos distúrbios benignos apresentam tal sintoma. O sangramento pós-menopausa leva à alta suspeição, com probabilidade de 5 a $10 \%$ de diagnóstico de carcinoma endometrial (GREDMARK et al., 1995). Pacientes com doença avançada podem ter sinais e sintomas semelhantes aos observados em casos de câncer de ovário avançado, como dor abdominal ou pélvica, distensão abdominal, saciedade precoce ou alterações intestinais ou urinárias (BURKE et al., 2014).

A ultrassonografia transvaginal (USTV) deve ser usada como uma ferramenta de diagnóstico pré-operatório, podendo fornecer ao cirurgião informações adicionais importantes para a escolha do procedimento cirúrgico, mas não há valor de corte para a espessura endometrial que alie alta sensibilidade com baixos índices falso-positivos (SCHRAMM et al., 2017). Um comitê recente do American College of Obstetricians and Gynecologists (ACOG) indica que o valor de corte para a medida do endométrio no USTV deve ser de $4 \mathrm{~mm}$ ou menos. Logo, pacientes na pós-menopausa com espessura endometrial maior que $5 \mathrm{~mm}$ devem ser avaliadas com amostra tecidual, especialmente se houver sangramento. O ponto de corte ideal para avaliar mulheres na pré-menopausa ainda não foi definido, mas as recomendações incluem a medida de $16 \mathrm{~mm}$ ou menos como dentro da normalidade. Em todos os pacientes, se o sangramento persiste apesar de USTV normal, a biópsia endometrial deverá ser realizada (BRAUN, 2016). Alguns estudos sugerem que a USTV, realizada por profissional experiente, apresenta precisão semelhante à da ressonância magnética $(\mathrm{RM})$ para avaliação de invasão miometrial e cervical, com a vantagem de apresentar custo reduzido. Entretanto, para adequada avaliação linfonodal, a RM é preferível (MORICE et al., 2016).

O procedimento mais comum para amostragem endometrial ambulatorial é a biópsia com cateter de Pipelle. A histeroscopia com biópsia continua sendo o padrão ouro na avaliação diagnóstica do câncer endometrial, com melhor acurácia diagnóstica quando comparada à dilatação e curetagem (D \& C) (BURKE et al., 2014).

O único marcador tumoral clinicamente útil no manejo de pacientes com câncer endometrial é o CA-125, cuja dosagem elevada sugere a doença em estádios mais avançados (REIJNEN et al., 2019). 
A graduação histológica do câncer de endométrio deve ser determinada por avaliação microscópica, com análise do padrão de crescimento arquitetural do tumor. O sistema mais utilizado nessa classificação é o da Federação Internacional de Ginecologia e Obstetrícia (FIGO). As lesões de grau 1 geralmente são indolentes, com menor tendência à disseminação, as de grau 2 tem prognóstico intermediário e as de grau 3 tem maior potencial de invasão miometrial e metástase linfonodal (AMANT et al., 2005).

Os cânceres de endométrio podem disseminar-se de várias formas para além do útero. Os tumores endometriais do tipo I geralmente disseminam-se por contiguidade, via linfática, hematogênica ou peritoneal e os do tipo II tem padrão de disseminação semelhante aos dos carcinomas epiteliais do ovário. Os principais sítios de metástases incluem linfonodos pélvicos e para-aórticos, anexos e peritônio (WALKER et al., 2009).

$\mathrm{O}$ risco cumulativo estimado de câncer de endométrio é $0-96 \%$; o risco de mortalidade correspondente é de $0-23 \%$ e a taxa de mortalidade relacionada à incidência é de 0,24 , menor que a do câncer de mama $(0,32)$, câncer de ovário $(0,63)$ e câncer cervical $(0,55)$. A maioria dos casos de câncer do endométrio (75\%) são diagnosticados em estádio inicial (estádios FIGO I ou II), e a sobrevida global (SG) em 5 anos varia de 74 a 91\%. Para os tumores em estádio III da FIGO, a SG em 5 anos é de 57 a 66\% e para a doença em estádio IV da FIGO é de 20 a 26\%. Em relação à sobrevida livre de doença (SLD) em 5 anos, é estimada em 90\% dos pacientes sem metástase linfonodal, 60 a $70 \%$ naqueles com acometimento de linfonodos pélvicos e 30 a $40 \%$ em pacientes com acometimento de linfonodos para-aórticos. No entanto, uma substancial proporção de pacientes com câncer endometrial morre de outras patologias, pois esses pacientes frequentemente apresentam várias comorbidades (MORICE et al., 2016).

Nas últimas duas décadas diversos aprimoramentos têm sido adicionados ao manejo das neoplasias ginecológicas. Estas medidas referem-se praticamente a todas as etapas da atenção a paciente com neoplasias malignas, ou seja, aumento na cobertura dos rastreamentos, melhorias nos fluxos hierarquizados de atendimento e notáveis avanços no conhecimento da biologia tumoral, com consequentes modificações nos protocolos de tratamento (MORICE et al., 2016). Esta combinação de fatores tem permitido notáveis quedas na mortalidade por neoplasias.

A FIGO propôs, em 1958, as primeiras regras para classificação e estadiamento dos cânceres ginecológicos (MUTCH, 2009). No estadiamento do carcinoma endometrial, as classificações mais adotadas são as da FIGO de 2009 e a TNM, ambas baseadas no estadiamento cirúrgico, incluindo avaliação da extensão da invasão miometrial e doença 
metastática local e à distância (MORICE et al., 2016). As vantagens do estadiamento refletemse em melhor diagnóstico, avaliação prognóstica e melhor definição da terapia adjuvante (“Clinical Management Guidelines for Obstetrician-Gynecologists. Number 42, April”, 2003).

Em geral, as mulheres com câncer de endométrio devem ser submetidas à histerectomia, salpingooforectomia bilateral e estadiamento cirúrgico. As situações que podem contraindicar o tratamento cirúrgico primário são: desejo de preservação da fertilidade, alto grau de obesidade, alto risco operatório e doença clinicamente inoperável. A obesidade e outras comorbidades associadas como hipertensão, diabetes e doença pulmonar levam a estadiamento incompleto, aumento do tempo cirúrgico, aumento da perda sanguínea e infecção e deiscência de parede abdominal (EVERETT et al., 2003; FOLEY; LEE, 1990).

Os tumores do corpo uterino, cuja maioria é diagnosticada em estádios iniciais, apresentam a peculiaridade de coexistirem com diversas comorbidades. Na grande maioria dos casos o estadiamento e a abordagem terapêutica inicial requerem a realização de uma cirurgia. Historicamente, o estadiamento cirúrgico para o câncer de endométrio era realizado via laparotomia, incluindo histerectomia, salpingooforectomia bilateral, linfadenectomia pélvica e para-aórtica e coleta de citologia peritoneal (WALKER et al., 2009), mas a abordagem laparoscópica e robótica vem sendo cada vez mais empregada para os casos provavelmente restritos ao útero. O grau histológico na biópsia pré-operatória e a avaliação intraoperatória da profundidade de invasão miometrial foram os dois fatores mais utilizados pelos cirurgiões na determinação da necessidade de realização de dissecção de linfonodos pélvicos e para-aórticos, usualmente. Entretanto, estudos recentes questionam essa abordagem, devido à dificuldade em precisar essa avaliação (ELTABBAKH; SHAMONKI; MOUNT, 2005). Apesar da abordagem com histerectomia total simples (tipo I) ser suficiente para a maioria das mulheres, a histerectomia radical (tipo III) às vezes é feita em casos de invasão cervical ou na incerteza sobre a localização do tumor primário (endocervical ou endometrial). Omentectomia e biópsias peritoneais são comumente feitas em doenças de alto risco (MORICE et al., 2016).

A avaliação cirúrgica dos linfonodos para estadiamento na abordagem primária continua sendo um dos tópicos mais controversos em relação ao tratamento cirúrgico do câncer de endométrio, variando de nenhuma avaliação linfonodal, biópsia de linfonodo sentinela ou linfadenectomia pélvica e para-aórtica. A maioria dos cirurgiões concordam que a excisão ou biópsia de linfonodos suspeitos ou aumentados nas regiões pélvicas ou para-aórticas é importante para excluir acometimento metastático ganglionar. No entanto, ainda não há nenhuma evidência de ganho de sobrevida em pacientes submetidos à linfadenectomia, com 
clássico aumento em morbidade operatória, com possibilidade de 8 a $50 \%$ dos pacientes desenvolverem linfedema de membros inferiores, dependendo do número de linfonodos removidos, extensão da linfadenectomia e tratamento adjuvante proposto (MORICE et al., 2016).

A década de 1990 trouxe o uso de métodos cirúrgicos minimamente invasivos para replicar os objetivos tradicionais de cirurgia aberta, com aplicação no estadiamento do câncer endometrial. O Gynecologic Oncology Group (GOG) conduziu o estudo LAP2, ensaio randomizado multicêntrico com o objetivo de comparar laparoscopia e laparotomia no estadiamento cirúrgico do câncer endometrial, publicado em 2009, para tratamento de tumores com estadiamento clínico I e II (WALKER et al., 2009). No grupo operado por laparoscopia, observou-se taxas similares de lesões intraoperatórias, menos complicações moderadas a graves, menor período de internação e melhor qualidade de vida em 6 semanas de pósoperatório, porém associado a prolongamento no tempo cirúrgico. A conversão de laparoscopia para laparotomia ocorreu em $25,8 \%$ dos casos, principalmente por falta de exposição cirúrgica dependente do Índice de Massa Corporal (IMC). Observou-se taxas semelhantes, no seguimento de longo prazo, em relação à sobrevida e taxas de recidiva (MOORE; BREWER, 2017). O estudou LACE, divulgado em 2010, demonstrou que as pacientes submetidas ao tratamento com cirurgia laparoscópica apresentaram melhores resultados de qualidade de vida, tanto precoces quanto tardios (JANDA et al., 2010).

Em 2005, o Food and Drug Administration (FDA) liberou a plataforma para abordagem cirúrgica robótica, baseada no computador da Vinci, para tumores ginecológicos, adicionando outra ferramenta ao arsenal no manejo dos carcinomas endometriais, tendo sua segurança e viabilidade avaliadas retrospectiva e prospectivamente, em estudos multicêntricos e meta-análises (JOHNSON et al., 2017). Comparado com a abordagem laparoscópica, a taxa de complicações mais graves e número médio de linfonodos removidos foram semelhantes, entretanto com a abordagem robótica houve menor perda sanguínea (CARDENASGOICOECHEA et al., 2014).

As técnicas minimamente invasivas são efetivas e reduzem as complicações relacionadas à abordagem cirúrgica (TOZZI et al., 2005). Diversos estudos apontam vantagens da técnica laparoscópica sobre a laparotomia convencional. As principais vantagens em curto prazo referem-se a redução de dor e melhor qualidade de vida (JANDA et al., 2010; KORNBLITH et al., 2009; OBERMAIR et al., 2005). Alguns riscos também podem estar associados às técnicas laparoscópicas como possibilidade de lesão intraoperatória (vesical ou 
intestinal) em pacientes obesas ou o implante de tumor em sítios de punção (TANAKA et al., 2017). Poucos estudos investigam os efeitos de longo prazo do uso de técnicas minimamente invasivas. As evidências apontam no sentido de equivalência em termos de sobrevida em longo prazo entre a laparotomia e a laparoscopia (GALAAL et al., 2012; JANDA et al., 2017), com benefícios de curto prazo para a técnica minimamente invasiva.

A maioria das pacientes tratadas cirurgicamente pode ser apenas monitorada clinicamente a cada três ou quatro meses nos dois primeiros anos de seguimento, com exame pélvico detalhado, e a cada seis meses por mais três anos, antes de retomar consultas ginecológicas anuais (KOH; DORIGO; MUTCH, 2017). Para tumores avançados, exames de imagem e dosagem de CA-125 podem ser úteis no seguimento e monitorização pós tratamento (BURKE et al., 2014).

O tratamento adjuvante poderá utilizar radioterapia, tratamento hormonal e quimioterapia, a depender do estádio clínico da doença (JOHNSON et al., 2017). As recomendações de tratamento adjuvante baseiam-se no tipo histológico, grau nuclear, profundidade da invasão miometrial e cervical, invasão do espaço linfovascular, citologia peritoneal, e estágio da doença, com um esforço para evitar a toxicidade do tratamento excessivo (WALKER et al., 2009).

A quimioterapia com paclitaxel, doxorrubicina e cisplatina é o tratamento adjuvante de escolha para o câncer de endométrio em estádio avançado, após a cirurgia. Há evidências moderadas de que a quimioterapia aumenta o tempo de sobrevida após a cirurgia primária em aproximadamente 25\%, em relação à radioterapia, no câncer endometrial nos estágios III e IV, com evidências limitadas de que está associado a mais efeitos adversos. Existe alguma incerteza quanto ao fato de os regimes triplos oferecerem benefícios de sobrevivência semelhantes aos regimes duplos, a longo prazo. Mais pesquisas são necessárias para determinar qual regime de quimioterapia é o mais eficaz e o menos tóxico, e se a adição de radioterapia melhora ainda mais os resultados (GALAAL et al., 2014).

Tradicionalmente, pacientes que apresentavam risco de recorrência pós-operatória após tratamento do câncer de endométrio, eram conduzidas à radioterapia. A radioterapia como terapia adjuvante no estádio I tem papel controverso, devido à baixa taxa de recidiva e falta de estudos randomizados, toxicidade associada, além de não apresentar impacto na SG (CREUTZBERG et al., 2000). No ensaio do GOG, observou-se que ocorreu redução evidente no risco de recorrência no subgrupo de mulheres com risco intermediário ou alto, com três fatores de risco (tumores grau 2 ou 3, invasão angiolinfática e invasão do terço externo do 
miométrio) (KEYS et al., 2004). No estudo PORTEC-1, pacientes com adenocarcinoma de endométrio estádio I, graus 1 e 2, com invasão de mais de 50\% do miométrio, ou graus 2 e 3 , com invasão igual ou menor que 50\%, submetidas à cirurgia radical, foram randomizadas para tratamento da pelve com radioterapia vs. observação. Os resultados mostraram aumento significativo no controle local em cinco ano, porém com aumento de toxicidade gastrintestinal, sem benefício em termos de sobrevida (CREUTZBERG et al., 2011). Para pacientes com estádio cirúrgico II, a indicação de radioterapia é ainda mais controversa, devendo ser abordada individualmente e com base na análise dos fatores de risco concomitantes (LESTER-COLL et al., 2017). O estudo randomizado PORTEC-2 comparou a eficácia e a qualidade de vida das pacientes tratadas com radioterapia externa vs. braquiterapia de fundo vaginal, com equivalência nos dois grupos para recorrência vaginal, sem diferença em termos de sobrevida (NOUT et al., 2010).

A utilização de terapia hormonal no câncer endometrial, com progesterona, pode ser utilizada como tratamento primário em pacientes com risco cirúrgico extremamente alto. No entanto, o valor preditivo do status do receptor de estrogênio e de progesterona permanece um pouco controverso. Relatório preliminar do estudo PARAGON (ANZGOG 0903), que observou cânceres endometriais recorrentes, com expressão positiva para esses receptores, mostrou que as pacientes apresentaram benefício clínico de $44 \%$ em 3 meses com uso do anastrozol, com melhora significativa na qualidade de vida (LEE; LHEUREUX; OZA, 2017).

Muitos fatores clínicos e patológicos influenciam a probabilidade de recidiva de câncer de endométrio e a sobrevida, mas o estadiamento cirúrgico da FIGO é a principal variável, uma vez que engloba diversos fatores de risco importantes (MOORE; BREWER, 2017).

Nas últimas duas décadas ampliou-se de forma substancial o uso de técnicas minimamente invasivas como a videolaparoscopia e mais recentemente a cirurgia robótica para a cirurgia do câncer de endométrio (WALKER et al., 2009). Estudar coortes de seguimento onde ambos os métodos foram aplicados concomitantemente na prática real é fundamental para se estimar os efeitos populacionais da implantação das técnicas minimamente invasivas fora de estudos clínicos randomizados. 
2 Objetivos 


\subsection{Objetivo geral}

Comparar as técnicas laparotômica e laparoscópica em uma coorte de tumores de endométrio.

\subsection{Objetivos específicos}

- Comparar os parâmetros intraoperatórios das técnicas laparoscópicas e laparotômicas para o estadiamento cirúrgico do câncer de endométrio.

- Comparar resultados de curto prazo das técnicas laparoscópicas e laparotômicas para o estadiamento cirúrgico do câncer de endométrio.

- Avaliar o risco de recorrência de médio e longo prazo em mulheres submetidas a laparoscopia e laparotomia para o câncer de endométrio.

- Avaliar a sobrevida em mulheres submetidas a laparoscopia e laparotomia para o câncer de endométrio. 
3 Artigo Submetido 


\section{Title page}

\section{Title}

Comparison of laparoscopy and laparotomy for early-stage endometrial cancer

\section{Author names and affiliations.}

Licerio Miguel ${ }^{1}$

Christiani Bisinoto de Sousa ${ }^{2}$

Julio Cesar Rosa e Silva ${ }^{1}$

Omero Benedito Poli Neto ${ }^{1}$

Daniel Guimarães Tiezzi ${ }^{1}$

Jurandyr Moreira de Andrade ${ }^{2}$

Francisco Jose Candido dos Reis ${ }^{1}$

${ }^{1}$ Postgraduate Gynaecology and Obstetrics Program, Department of Gynaecology and Obstetrics, Ribeirao Preto Medical School, University of Sao Paulo, Ribeirao Preto.

${ }^{2}$ Department of Gynaecology and Obstetrics, Ribeirao Preto Medical School, University of Sao Paulo, Ribeirao Preto.

\section{Corresponding author.}

Francisco José Candido dos Reis. Departamento de Ginecologia e Obstetrícia. Av. Bandeirantes 3900, 8o andar, Ribeirao Preto, Brazil, 14049-900. Fax number: 5516 36021524. Telephone number: 5516 36022589. E-mail address: fjcreis@usp.br 


\section{Highlights}

- Laparoscopic surgery is associated with less postoperative surgical complications in women with endometrial cancer.

- There is no increased risk of endometrial cancer recurrence associated with laparoscopic surgery.

- Laparoscopic surgery is associated with improved overall survival in women with endometrial cancer. 


\section{Abstract \\ Introduction.}

The surgical approach is a fundamental component of early endometrial cancer management. Because of comorbidities associated with the disease, complications of surgery are common. Laparoscopic surgery may reduce surgical complications but also have risks.

\section{Objective.}

Our aim was to compare the short and long-term outcomes of laparoscopy and laparotomy in early endometrial cancer.

\section{Methods.}

Our cohort included all women undergone surgery for presumed early endometrial cancer at the Clinics Hospital of Ribeirão Preto Medical School, from January 1998 to December 2017. The type of surgery was decided according to surgeons' preference. We compared the characteristics of patients submitted to each type of surgery, short term outcomes, disease-free survival, and overall survival.

\section{Results.}

From a total of 400 women were included in this study, 316 undergone to laparotomy and 84 to laparoscopy. Women submitted to laparotomy presented higher frequency of grade 3 tumors (22.8\% vs. $11.9 \% ; \mathrm{P}=0.006)$ and higher frequency of adjuvant chemotherapy (14.6\% vs. $6 \%$; $\mathrm{P}=0.055)$. Laparoscopy was associated with increased surgical time ((194.7 min vs. $165.1 \mathrm{~min}$; $\mathrm{P}<0.001)$, and reduced rate of surgical complications (0 vs. 9.2\%; $\mathrm{P}=0.008)$. Laparoscopy did not increase the risk of recurrence (HR: $0.43,95 \% \mathrm{CI} 0.18-1.02 ; \mathrm{P}=0.06$ ) and increased the ten-years overall survival (HR: $0.44,95 \%$ CI $0.22-0.91 ; \mathrm{P}=0.03$ )

\section{Conclusion.}

Laparoscopy presented clear advantages over laparotomy for early endometrial cancer. It was associated with a lower rate of surgical complications and better overall survival.

\section{Keywords.}

Endometrial carcinoma; laparoscopy; laparotomy; survival. 


\section{Background}

Uterine corpus cancer is the most common among women in the United States, accounting for $7 \%$ of all female malignant tumors, and for $4 \%$ of estimated cancer deaths [1]. Among the uterine corpus tumors, endometrial carcinoma corresponds to 97\% [2]. It is associated with well-defined risk factors such as obesity, nulliparity or genetic risk such as Lynch syndrome [3]. Compared to other female tumors, endometrial carcinoma has good prognosis. Its mortality-to-incidence ratio is 0.24 , that is lower than that of breast cancer $(0.32)$, ovarian cancer (0.63), and cervical cancer (0.55) [4]. However, endometrial carcinoma frequently coexists with clinical comorbidities. Endometrial tumors are significantly more frequent in obese women [5]. Obesity and other associated comorbidities such as hypertension, diabetes, and pulmonary disease lead to incomplete staging, increased surgical time, increased blood loss, and abdominal wall infection and dehiscence [6,7].

Over the past two decades, the use of laparoscopy and more recently, robotic surgery for endometrial cancer surgery has expanded substantially. Minimally invasive techniques are effective and reduce complications related to the surgical approach [8]. Several studies showed advantages of the laparoscopic technique over conventional laparotomy. The main short-term advantages relate to pain reduction and better postoperative quality of life [9-11]. Some risks like urinary tract or intestinal injury and tumor dissemination may also be associated with minimally invasive techniques. Few studies investigated the long-term outcomes and current evidence points to equivalence between conventional laparotomy and laparoscopic surgery $[12,13]$.

The primary objective was to compare laparoscopy with laparotomy with regard to overall survival and disease-free survival. Secondary objectives included comparing the two groups with regard to intraoperative and postoperative outcomes. 


\section{Methods}

\section{Study design}

The retrospective cohort included all women treated for clinical early endometrial cancer at Clinics Hospital of Ribeirão Preto Medical School from 1998 to 2017. The Institutional Board for Ethics in Research approved the study (CAAE: 81115817.5.0000.5440), and the informed consent waived.

\section{Inclusion and exclusion criteria}

Patients were eligible if they had clinical endometrial cancer without clinical or image evidence of cervical or extrauterine disease. Exclusion criteria were: advanced disease at diagnosis, previous surgical treatment for endometrial cancer, surgery not performed for other reason (eg, impaired performance status), previous chemotherapy or radiotherapy for endometrial cancer treatment.

\section{Surgical procedures}

During this period, the decision of whether to use laparoscopic surgery or traditional laparotomy followed the surgeon preference. For laparoscopic surgeries, we used umbilical Veress needle entry in all cases, $\mathrm{CO} 2$ for abdominal distention and Trendelenburg position. Bipolar electrical devicer were used for vessel sealing. Vaginal approaches were used for colpotomy and section of cardinal/uterosacral ligaments. Midline incisions were used as preferential route for laparotomies. In both types of surgeries, the decision on when to perform lymphadenectomy was based on preoperative image and biopsy (histology and grade) and intraoperative findings (estimation of myometrial invasion). As a general rule, women with grade I/II superficial endometrioid tumors did not undergo lymphadenectomy.

\section{Variables and Statistical Analysis}

The variables collected were: type of surgery, age at diagnosis, body index mass (BMI), sonographic uterine volume, tumor histology, tumor FIGO stage, tumor grade, preoperative CA 125 levels, adjuvant treatment (radiotherapy, chemotherapy), duration of anesthesia, duration of surgery, lymphadenectomy, intraoperative complications, surgical postoperative complications, clinical postoperative complications, thromboembolic events, blood transfusion needed, hospital postoperative stay, ten-years disease-free survival and tens-years overall survival. 
Disease-free survival (DFS) was defined as the interval from the time of primary surgery to any documented disease recurrence. Overall survival (OS) was defined as the interval from primary surgery to death by any cause. For patients who were still alive at the time of analysis, DFS and OS were censored at the date of last follow up.

Summary statistics were used to compare the variables from women submitted to laparoscopy and laparotomy. To deal with missing values for preoperative CA125, BMI, and uterine volume, we used multiple imputations [14]. Qualitative variables are displayed using frequencies and percentages. Kaplan-Meier product-limit estimator and Cox proportional hazards regression were used for survival analysis. Data manipulation and statistical analysis were performed with R Studio Version 1.2.1335. 


\section{Results}

From 1998 to 2017, 507 cases of endometrial cancer were identified. A total of 107 cases were excluded due to the following reasons: 43 were advanced at diagnosis, 8 presented another concomitant primary tumor, 11 were submitted to vaginal surgery, 34 were not submitted to surgical staging at our hospital, and nine did not have recorded follow up. The remaining 400 women with presumed early endometrial cancer were included in this study. Three hundred sixteen were submitted to conventional laparotomy and 84 to laparoscopic surgery.

There were 55 missing values for BMI, 48 for uterine volume and 82 for preoperative CA 125, therefore missing data were imputed for the analysis. The characteristics of the included patients were presented in Table 1. The two groups were well balanced according to most of variables. Women submitted to laparotomy presented higher frequency of grade 3 tumors (22.8\% vs. $11.9 \% ; \mathrm{P}=0.006)$ and higher frequency of adjuvant chemotherapy (14.6\% vs. $6 \%$; $\mathrm{P}=0.055)$.

In Table 2 are presented the surgical outcomes associated with each type of intervention. Both groups presented similar results on the percentage of women submitted to lymphadenectomy, the number of nodes removed, intraoperative complications, postoperative clinical complications, thromboembolic events, blood transfusion, and postoperative hospital stay. We observed increased anesthesia time (233.5 min vs. $201.5 \mathrm{~min} ; \mathrm{P}<0.001)$ and surgical time (194.7 min vs. $165.1 \mathrm{~min} ; \mathrm{P}<0.001)$ for surgeries performed by laparoscopy. Laparoscopy was associated with lower rate of surgical complications than laparotomy $(9.2 \%$ vs. $0 ; \mathrm{P}=0.008)$. Among the 29 cases of surgically related complications: ten were wound dehiscence, ten wound evisceration, three ileuses, three bowel obstruction, and three abdominal sepsis. Considering all 54 cases of postoperative complications, the adjusted multivariate logistic regression identified laparoscopic surgery as protective variable $(\mathrm{OR}=0.33 ; 95 \% \mathrm{CI}$ : $0.13-0.88 ; \mathrm{P}=0.016)$, and age as risk variable $(\mathrm{OR}=1.04 ; 95 \% \mathrm{CI}: 1.01-1.08 ; \mathrm{P}=0.012)$.

We observed a long-term benefit of laparoscopic surgery in terms of ten years of overall mortality (Figure 1). Table 3 shows the hazard ratio associated with several prognostic variables and the independent benefit of the laparoscopic approach is confirmed (HR: 0.44, 95\% CI 0.22-0.91; $\mathrm{P}=0.03)$. Other independent risk factors for all-cause mortality were age at diagnosis (HR: 1.06, 95\% CI 1.03-1.08; P < 0.0001) and the occurrence of postoperative complications (HR: 1,98, 95\% CI 1.08-3.62; $\mathrm{P}=0.03$ ). On the other hand, as shown in Table 
4, laparoscopic surgery was not associated with risk of tumor recurrence (HR: $0.43,95 \%$ CI $0.18-1.02 ; \mathrm{P}=0.06$ ). 


\section{Discussion}

Our results show an important benefit of laparoscopic surgery for early endometrial carcinoma management. Although there was an increment of 30 minutes on the time of surgery and anesthesia, the risk of surgical complications was significantly reduced. The laparoscopic surgery was not associated with risk of tumor relapse and resulted in improvement of tens-years overall survival.

The main strengths of our study were: a well-documented cohort, the follow up was rigorous, and laparoscopy and laparotomy were performed in concomitance over time. The follow up allowed a long-term observation of outcomes and the concomitance of procedures was important to minimize the bias associated with trends in survival associated with improvements in treatment quality [15]. Our study design also allowed the comparison of the effectiveness of both procedures in a real-world setting [16]. One limitation of our study was the fact that laparoscopy or laparotomy was performed according to surgeon preference, and therefore the procedures were conducted by different surgeons that might have different skills and experience.

Compared with laparotomy, laparoscopy was associated with fewer complications. Our results are concordant with others in demonstrating a reduced risk of surgical complications in patients who underwent laparoscopic surgery, as well as the association of laparoscopy with a significantly longer operative time $[17,18]$. However, there are also clinical trials that did not find differences in surgical complications between laparotomy and laparoscopy [19]. A metaanalysis of data from eight randomized, controlled trials (RCTs) found similar intra-operative complication rates in both surgical approaches, less blood loss in laparoscopy, shorter operative time in laparotomy, and significant advantage of laparoscopy over laparotomy in terms of postoperative complications [20]. Similarly to others [8], we found that the surgical technique was the most important variable significantly associated with complication rate.

There are few studies on long term outcomes of laparoscopic surgery published. We did not find evidence increment of risk of recurrence associated with laparoscopic surgery. This result is similar to a previous metanalysis [21]. The LAP2 trial also confirmed, with a median of five years of follow-up, the safety of laparoscopy in terms of recurrence and survival [22]. On the other hand, we found a significant improvement in the ten-years overall survival associated with laparoscopic surgery. Because early endometrial cancer has a high probability of cure with surgical treatment, the main causes of death among these patients are not endometrial cancer 
itself. The main cause of death is cardiovascular disease [23,24]. Laparoscopic surgery is associated with fewer surgically related complications and better postoperative quality of life $[10,25]$ which may be associated with improved posttreatment general health conditions and reduced all-cause mortality.

In conclusion, our results confirm that laparoscopy was effective and safe for early endometrial cancer management. Laparoscopic surgery did not increase the risk of cancer recurrence and was associated with fewer surgically related complications. In our study, laparoscopic surgery was also associated with better ten-year overall survival.

\section{Role of the funding source}

F. J. Candido dos Reis (grant number 303210/2018-4) was funded by the National Council for Scientific and Technological Development (CNPq)

This study was financed in part by the Coordenação de Aperfeiçoamento de Pessoal de Nível Superior - Brasil (CAPES) - Finance Code 001

\section{Author contributions}

Conceived and designed the study: L.M, and F.J.C.R.

Collected and analyzed data: L.M, C.B.S., J.C.R.S., and F.J.C.R.

Helped with writing first draft of manuscript: All authors.

Provided critical insight and revision to manuscript: All authors.

\section{Declaration of competing interest}

All authors report no conflicts of interest to disclose. 


\section{References}

[1] R.L. Siegel, K.D. Miller, A. Jemal, Cancer statistics, 2019, CA Cancer J Clin. 69 (2019) 7-34. https://doi.org/10.3322/caac.21551.

[2] P.G. Rose, Endometrial Carcinoma, New England Journal of Medicine. 335 (1996) 640-649. https://doi.org/10.1056/NEJM199608293350907.

[3] P. Morice, A. Leary, C. Creutzberg, N. Abu-Rustum, E. Darai, Endometrial cancer, Lancet. 387 (2016) 1094-1108. https://doi.org/10.1016/S0140-6736(15)00130-0.

[4] J. Ferlay, I. Soerjomataram, R. Dikshit, S. Eser, C. Mathers, M. Rebelo, D.M. Parkin, D. Forman, F. Bray, Cancer incidence and mortality worldwide: sources, methods and major patterns in GLOBOCAN 2012, Int J Cancer. 136 (2015) E359-86.

https://doi.org/10.1002/ijc.29210.

[5] A. Bergström, P. Pisani, V. Tenet, A. Wolk, H.O. Adami, Overweight as an avoidable cause of cancer in Europe, Int. J. Cancer. 91 (2001) 421-430. https://doi.org/10.1002/10970215(200002)9999:9999<::aid-ijc1053>3.0.co;2-t.

[6] E. Everett, H. Tamimi, B. Greer, E. Swisher, P. Paley, L. Mandel, B. Goff, The effect of body mass index on clinical/pathologic features, surgical morbidity, and outcome in patients with endometrial cancer, Gynecol. Oncol. 90 (2003) 150-157. https://doi.org/10.1016/s0090-8258(03)00232-4.

[7] K. Foley, R.B. Lee, Surgical complications of obese patients with endometrial carcinoma, Gynecol. Oncol. 39 (1990) 171-174. https://doi.org/10.1016/00908258(90)90427-m.

[8] R. Tozzi, S. Malur, C. Koehler, A. Schneider, Laparoscopy versus laparotomy in endometrial cancer: first analysis of survival of a randomized prospective study, J Minim Invasive Gynecol. 12 (2005) 130-136. https://doi.org/10.1016/j.jmig.2005.01.021.

[9] M. Janda, V. Gebski, A. Brand, R. Hogg, T.W. Jobling, R. Land, T. Manolitsas, A. McCartney, M. Nascimento, D. Neesham, J.L. Nicklin, M.K. Oehler, G. Otton, L. Perrin, S. Salfinger, I. Hammond, Y. Leung, T. Walsh, P. Sykes, H. Ngan, A. Garrett, M. Laney, T.Y. Ng, K. Tam, K. Chan, C.D.H. Wrede, S. Pather, B. Simcock, R. Farrell, A. Obermair, Quality of life after total laparoscopic hysterectomy versus total abdominal hysterectomy for stage I endometrial cancer (LACE): a randomised trial, Lancet Oncol. 11 (2010) 772-780. https://doi.org/10.1016/S1470-2045(10)70145-5. 
[10] A.B. Kornblith, H.Q. Huang, J.L. Walker, N.M. Spirtos, J. Rotmensch, D. Cella, Quality of life of patients with endometrial cancer undergoing laparoscopic international federation of gynecology and obstetrics staging compared with laparotomy: a Gynecologic Oncology Group study, J. Clin. Oncol. 27 (2009) 5337-5342.

https://doi.org/10.1200/JCO.2009.22.3529.

[11] A. Obermair, T.P. Manolitsas, Y. Leung, I.G. Hammond, A.J. McCartney, Total laparoscopic hysterectomy versus total abdominal hysterectomy for obese women with endometrial cancer, Int. J. Gynecol. Cancer. 15 (2005) 319-324. https://doi.org/10.1111/j.1525-1438.2005.15223.x.

[12] K. Galaal, A. Bryant, A.D. Fisher, M. Al-Khaduri, F. Kew, A.D. Lopes, Laparoscopy versus laparotomy for the management of early stage endometrial cancer, Cochrane Database Syst Rev. (2012) CD006655. https://doi.org/10.1002/14651858.CD006655.pub2.

[13] M. Janda, V. Gebski, L.C. Davies, P. Forder, A. Brand, R. Hogg, T.W. Jobling, R. Land, T. Manolitsas, M. Nascimento, D. Neesham, J.L. Nicklin, M.K. Oehler, G. Otton, L. Perrin, S. Salfinger, I. Hammond, Y. Leung, P. Sykes, H. Ngan, A. Garrett, M. Laney, T.Y. Ng, K. Tam, K. Chan, C.D. Wrede, S. Pather, B. Simcock, R. Farrell, G. Robertson, G. Walker, N.R. Armfield, N. Graves, A.J. McCartney, A. Obermair, Effect of Total Laparoscopic Hysterectomy vs Total Abdominal Hysterectomy on Disease-Free Survival Among Women With Stage I Endometrial Cancer: A Randomized Clinical Trial, JAMA. 317 (2017) 1224-1233. https://doi.org/10.1001/jama.2017.2068.

[14] I.R. White, P. Royston, A.M. Wood, Multiple imputation using chained equations: Issues and guidance for practice, Stat Med. 30 (2011) 377-399. https://doi.org/10.1002/sim.4067.

[15] S. Inoue, S. Hosono, H. Ito, I. Oze, Y. Nishino, M. Hattori, T. Matsuda, I. Miyashiro, T. Nakayama, M. Mizuno, K. Matsuo, K. Kato, H. Tanaka, Y. Ito, Improvement in 5-Year Relative Survival in Cancer of the Corpus Uteri From 1993-2000 to 2001-2006 in Japan, J Epidemiol. 28 (2018) 75-80. https://doi.org/10.2188/jea.JE20170008.

[16] H.-S. Kim, S. Lee, J.H. Kim, Real-world Evidence versus Randomized Controlled Trial: Clinical Research Based on Electronic Medical Records, J Korean Med Sci. 33 (2018). https://doi.org/10.3346/jkms.2018.33.e213. 
[17] J.L. Walker, M.R. Piedmonte, N.M. Spirtos, S.M. Eisenkop, J.B. Schlaerth, R.S. Mannel, G. Spiegel, R. Barakat, M.L. Pearl, S.K. Sharma, Laparoscopy Compared With Laparotomy for Comprehensive Surgical Staging of Uterine Cancer: Gynecologic Oncology Group Study LAP2, JCO. 27 (2009) 5331-5336. https://doi.org/10.1200/JCO.2009.22.3248.

[18] J.D. Wright, A.I. Neugut, E.T. Wilde, D.L. Buono, W.-Y. Tsai, D.L. Hershman, Use and Benefits of Laparoscopic Hysterectomy for Stage I Endometrial Cancer Among Medicare Beneficiaries, JOP. 8 (2012) e89-e99. https://doi.org/10.1200/JOP.2011.000484.

[19] M.J.E. Mourits, C.B. Bijen, H.J. Arts, H.G. ter Brugge, R. van der Sijde, L. Paulsen, J. Wijma, M.Y. Bongers, W.J. Post, A.G. van der Zee, G.H. de Bock, Safety of laparoscopy versus laparotomy in early-stage endometrial cancer: a randomised trial, Lancet Oncol. 11 (2010) 763-771. https://doi.org/10.1016/S1470-2045(10)70143-1.

[20] F. Zullo, A. Falbo, S. Palomba, Safety of laparoscopy vs laparotomy in the surgical staging of endometrial cancer: a systematic review and metaanalysis of randomized controlled trials, Am. J. Obstet. Gynecol. 207 (2012) 94-100. https://doi.org/10.1016/j.ajog.2012.01.010.

[21] S. Palomba, A. Falbo, T. Russo, F. Zullo, Updating of a recent meta-analysis of randomized controlled trials to assess the safety and the efficacy of the laparoscopic surgery for treating early stage endometrial cancer, Gynecol. Oncol. 114 (2009) 135-136. https://doi.org/10.1016/j.ygyno.2009.03.025.

[22] J.L. Walker, M.R. Piedmonte, N.M. Spirtos, S.M. Eisenkop, J.B. Schlaerth, R.S. Mannel, R. Barakat, M.L. Pearl, S.K. Sharma, Recurrence and Survival After Random Assignment to Laparoscopy Versus Laparotomy for Comprehensive Surgical Staging of Uterine Cancer: Gynecologic Oncology Group LAP2 Study, JCO. 30 (2012) 695-700. https://doi.org/10.1200/JCO.2011.38.8645.

[23] A.S. Felix, J.K. Bower, R.M. Pfeiffer, S.V. Raman, D.E. Cohn, M.E. Sherman, High cardiovascular disease mortality after endometrial cancer diagnosis: Results from the Surveillance, Epidemiology, and End Results (SEER) Database, International Journal of Cancer. 140 (2017) 555-564. https://doi.org/10.1002/ijc.30470.

[24] K.K. Ward, N.R. Shah, C.C. Saenz, M.T. McHale, E.A. Alvarez, S.C. Plaxe, Cardiovascular disease is the leading cause of death among endometrial cancer patients, Gynecol. Oncol. 126 (2012) 176-179. https://doi.org/10.1016/j.ygyno.2012.04.013. 
[25] F. Zullo, S. Palomba, T. Russo, A. Falbo, M. Costantino, A. Tolino, E. Zupi, P. Tagliaferri, S. Venuta, A prospective randomized comparison between laparoscopic and laparotomic approaches in women with early stage endometrial cancer: a focus on the quality of life, Am. J. Obstet. Gynecol. 193 (2005) 1344-1352.

https://doi.org/10.1016/j.ajog.2005.02.131. 
Table 1. Characteristics of patients

\begin{tabular}{|c|c|c|c|}
\hline Variable & Laparotomy & Laparoscopy & $\mathrm{P}$ \\
\hline Cases & $\mathrm{n}=316$ & $\mathrm{n}=84$ & \\
\hline Age (years) & $64.6(10.3)$ & $64.2(11.6)$ & 0.763 \\
\hline Body mass index (kg/m2) & $31.1(6.9)$ & $33.1(8.7)$ & 0.064 \\
\hline Uterine volume (cm3) & $118.3(96.7)$ & $108.3(79.8)$ & 0.384 \\
\hline Hystologic type & & & 0.422 \\
\hline Endometrioid & $271(85.8 \%)$ & $78(92.9 \%)$ & \\
\hline Serous & $12(3.8 \%)$ & $2(2.4 \%)$ & \\
\hline Clear cell & $3(0.9 \%)$ & $0(0 \%)$ & \\
\hline Mixed cell & $9(2.8 \%)$ & $1(1.2 \%)$ & \\
\hline Carcinossarcoma & $11(3.5 \%)$ & $3(3.6 \%)$ & \\
\hline Undifferentiated & $10(3.2 \%)$ & $0(0 \%)$ & \\
\hline FIGO stage & & & 0.508 \\
\hline IA & $134(42.4 \%)$ & $39(46.4 \%)$ & \\
\hline IB & $98(31 \%)$ & $27(32.1 \%)$ & \\
\hline II & $39(12.3 \%)$ & $9(10.7 \%)$ & \\
\hline IIIA & $18(5.7 \%)$ & $5(6 \%)$ & \\
\hline IIIB & $3(0.9 \%)$ & $2(2.4 \%)$ & \\
\hline IIIC & $24(7.6 \%)$ & $2(2.4 \%)$ & \\
\hline Grade & & & 0.006 \\
\hline I & $102(32.3 \%)$ & $42(50 \%)$ & \\
\hline II & $142(44.9 \%)$ & $32(38.1 \%)$ & \\
\hline III & $72(22.8 \%)$ & $10(11.9 \%)$ & \\
\hline CA 125 (UI/ml) & $28.7(80.7)$ & $34.3(69.9)$ & 0.560 \\
\hline Radiotherapy & & & 0.134 \\
\hline None & $170(53.8 \%)$ & $53(63.1 \%)$ & \\
\hline External & $23(7.3 \%)$ & $9(10.7 \%)$ & \\
\hline Brachitherapy & $27(8.5 \%)$ & $3(3.6 \%)$ & \\
\hline Combined & $96(30.4 \%)$ & $19(22.6 \%)$ & \\
\hline Adjuvant chemotherapy & $46(14.6 \%)$ & $5(6 \%)$ & 0.055 \\
\hline
\end{tabular}


Table 2. Results according to the type of surgery.

\begin{tabular}{lccc}
\hline Variable & Laparotomy & Laparoscopy & $\mathrm{P}$ \\
\hline Cases & $\mathrm{n}=316$ & $\mathrm{n}=84$ & \\
Duration of anaesthesia (min) & $201.5(55.8)$ & $233.5(58.5)$ & $<0.001$ \\
Duration of surgery (min) & $165.1(52.7)$ & $194.7(55.7)$ & $<0.001$ \\
Lymphadenectomy & & & 0.525 \\
$\quad$ No & $82(25.9 \%)$ & $20(23.8 \%)$ & \\
$\quad$ Pelvic & $230(72.8 \%)$ & $64(76.2 \%)$ & \\
$\quad$ Pelvic and aortic & $4(1.3 \%)$ & $0(0 \%)$ & \\
Number of nodes & $8.0[7.0]$ & $7.0[8.8]$ & 0.505 \\
Intraoperative complication & $13(4.1 \%)$ & $3(3.6 \%)$ & 1.000 \\
Surgical postoperative complication & $29(9.2 \%)$ & $0(0 \%)$ & 0.008 \\
Clinical postoperative complication & $15(4.7 \%)$ & $5(6 \%)$ & 0.866 \\
Thromboembolic event & $5(1.6 \%)$ & $1(1.2 \%)$ & 1.000 \\
Blood transfusion & $18(5.7 \%)$ & $1(1.2 \%)$ & 0.151 \\
Postoperative hospital stay (days) & $2.0[1.0]$ & $2.0[0.0]$ & 0.176 \\
\hline
\end{tabular}


Table 3. Cox regression for all-cause mortality.

\begin{tabular}{|c|c|c|c|}
\hline Variable & HR & $95 \% \mathrm{CI}$ & $\mathrm{P}$ \\
\hline \multicolumn{4}{|l|}{ Surgery type } \\
\hline Laparotomy & Ref & & \\
\hline Laparoscopy & 0.44 & $(0.22-0.91)$ & 0.026 \\
\hline Age & 1.06 & $(1.03-1.08)$ & $<0.001$ \\
\hline \multicolumn{4}{|l|}{ Histology } \\
\hline Endometrioid & Ref & & \\
\hline Serous & 4.1 & $(1.79-9.37)$ & 0.001 \\
\hline Clear cell & 10.32 & $(0.99-107.29)$ & 0.051 \\
\hline Mixed cell & 0 & (0-Inf) & 0.996 \\
\hline Carcinossarcoma & 1.49 & $(0.49-4.53)$ & 0.480 \\
\hline Undifferentiated & 2.41 & $(0.64-9)$ & 0.192 \\
\hline \multicolumn{4}{|l|}{ Figo stage } \\
\hline IA & Ref & & \\
\hline IB & 1.09 & $(0.58-2.03)$ & 0.798 \\
\hline II & 1.17 & $(0.5-2.77)$ & 0.715 \\
\hline IIIA & 1.46 & $(0.5-4.29)$ & 0.490 \\
\hline IIIB & 5.42 & $(1.08-27.28)$ & 0.040 \\
\hline IIIC & 2.37 & $(0.83-6.78)$ & 0.107 \\
\hline \multicolumn{4}{|l|}{ Grade } \\
\hline G1 & Ref & & \\
\hline $\mathrm{G} 2$ & 1.41 & $(0.78-2.58)$ & 0.258 \\
\hline G3 & 1.67 & $(0.75-3.71)$ & 0.206 \\
\hline \multicolumn{4}{|l|}{ Radiotherapy } \\
\hline None & Ref & & \\
\hline External & 0.35 & $(0.08-1.44)$ & 0.144 \\
\hline Brachitherapy & 0.43 & $(0.1-1.86)$ & 0.259 \\
\hline External and Brachitherapy & 1.48 & $(0.77-2.86)$ & 0.242 \\
\hline Adjuvant chemotherapy & 0.72 & $(0.29-1.8)$ & 0.480 \\
\hline Postoperative complication & 1.98 & $(1.08-3.62)$ & 0.027 \\
\hline
\end{tabular}


Table 4. Cox regression for recurrence.

\begin{tabular}{lccc}
\hline Variable & HR & $95 \%$ CI & P \\
\hline $\begin{array}{l}\text { Surgery type } \\
\text { Laparotomy } \\
\quad \text { Laparoscopy }\end{array}$ & Ref & & \\
Age & 0.43 & $(0.18-1.02)$ & 0.057 \\
Histology & 1.02 & $(0.99-1.05)$ & 0.148 \\
$\quad$ Endometrioid & & & \\
Serous & Ref & & \\
Clear cell & 3.79 & $(1.28-11.26)$ & 0.017 \\
$\quad$ Mixed cell & 4.55 & $(0.48-43.6)$ & 0.189 \\
$\quad$ Carcinossarcoma & 1.04 & $(0.13-8.16)$ & 0.971 \\
$\quad$ Undifferentiated & 1.98 & $(0.6-6.5)$ & 0.261 \\
\end{tabular}

Figo stage

$\begin{array}{lccc}\text { IA } & \text { Ref } & & \\ \text { IB } & 2.94 & (1.3-6.65) & 0.010 \\ \text { II } & 4.55 & (1.65-12.54) & 0.003 \\ \text { IIIA } & 11.35 & (3.41-37.82) & <0.001 \\ \text { IIIB } & 33.68 & (6.85-165.5) & <0.001 \\ \text { IIIC } & 9.06 & (2.42-33.88) & 0.001\end{array}$

Grade

$\begin{array}{lccc}\text { G1 } & \text { Ref } & & \\ \text { G2 } & 3.23 & (1.3-8.04) & 0.012 \\ \text { G3 } & 6.32 & (2.15-18.57) & 0.001\end{array}$

Radiotherapy

None Ref

External $\quad 0.44 \quad(0.14-1.4) \quad 0.162$

$\begin{array}{llll}\text { Brachitherapy } & 0.98 \quad(0.32-3.04) & 0.975\end{array}$

External and Brachitherapy $\quad 0.75 \quad(0.35-1.6) \quad 0.458$

$\begin{array}{llll}\text { Chemotherapy } & 0.3 & (0.11-0.82) & 0.019\end{array}$

$\begin{array}{llll}\text { Postoperative complication } & 1.49 & (0.67-3.33) & 0.332\end{array}$ 


\section{Figure 1}

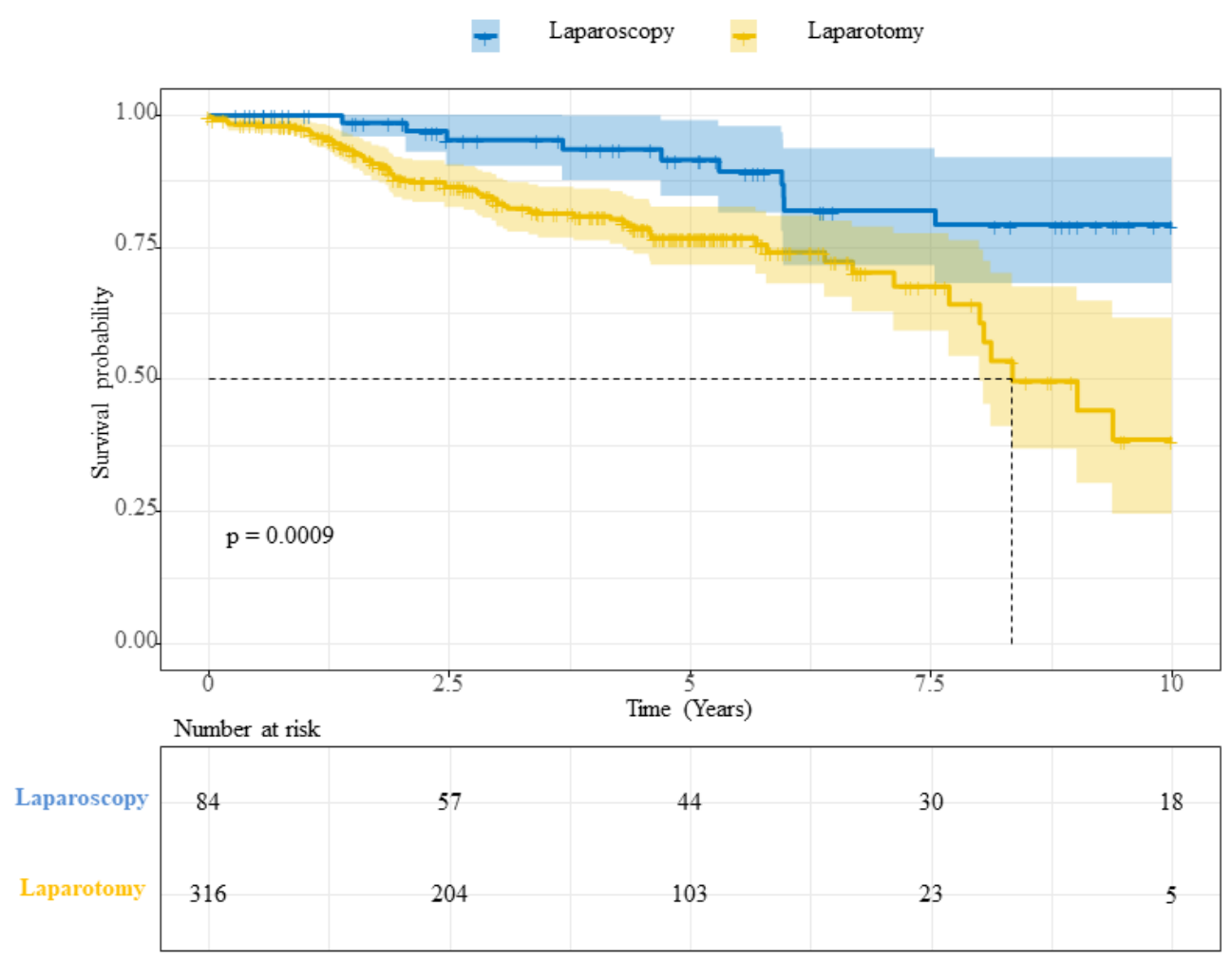

Figure 1. Kaplan-Meyer curve for ten-years survival of women with early endometrial cancer undergone laparoscopic surgery or conventional laparotomy. 


\section{Figura 2}

Gynecologic Oncology: Submission Confirmation

1 mensagem

Gynecologic Oncology <eesserver@eesmail.elsevier.com>

Responder a: Gynecologic Oncology <gyn@elsevier.com>

Para: fjcreis@usp.br

*** Automated email sent by the system ***

Title: Comparison of laparoscopy and laparotomy for early-stage endometrial cance

Authors: Licerio Miguel; Christiani B Sousa; Julio C Rosa e Silva; Omero B Poli Neto; Daniel G Tiezzi; Jurandyr M Andrade;

Research Paper

Dear Professor Candido-dos-Reis,

This is to confirm that the above-mentioned manuscript has been received for consideration in Gynecologic Oncology.

Your manuscript will undergo a quick screening process to ensure that it meets all submission requirements. Please note that if your manuscript does not meet all submission requirements, it will be returned to you without being seen by an editor or reviewers.

You will be able to check on the progress of your manuscript by logging on to the Elsevier Editorial System for Gynecologic Oncology as an author: hittps://ees.elsevier.com/ygyno/

Your paper will be given a manuscript number shortly and you will soon receive an e-mail with this number for your reference.

Thank you for submitting your manuscript to Gynecologic Oncology. Should you have any questions, please feel free to contact our office.

Kind regards,

Editorial Office

Gynecologic Oncology

E-mail: gyn@elsevier.com

For further assistance, please visit our customer support site at http://help.elsevier.com/app/answers/list/p/7923 Here you can search for solutions on a range of topics, find answers to frequently asked questions and learn more about EES via interactive tutorials. You will also find our $24 / 7$ support contact details should you need any further assistance from one of our customer support representatives. 
4 Conclusões 
- A laparoscopia foi eficaz e segura para o tratamento câncer inicial do endometrio.

- A cirurgia laparoscópica apresenta maior tempo cirúrgico em relação à cirurgia convencional.

- A cirurgia laparoscópica esta associada a redução substancial dos riscos de complicações cirúrgicas.

- A cirurgia laparoscópica não aumentou o risco de recorrência do câncer de endométrio.

- Em nosso estudo, a cirurgia laparoscópica também foi associada a uma melhor sobrevida global em dez anos. 
Anexos 


\section{ANEXO 1. Métodos Detalhados}

\section{Desenho do Estudo}

Trata-se de um estudo observacional populacional tipo coorte. Os sujeitos do estudo foram mulheres com confirmação histológica de tumores de endométrio. As participantes são originárias do serviço de oncologia ginecológica do Hospital das Clínicas da Faculdade de Medicina de Ribeirão Preto (HC FMRP-USP). O estudo foi aprovado pela Comissão de Ética e Pesquisa (CEP) do HC FMRP-USP (CAAE: 81115817.5.0000.5440), com concessão de Isenção do Termo de Consentimento Livre e Esclarecido (TCLE).

\section{Casuística}

Foram analisados 507 casos no período abrangido, com diagnóstico de câncer de endométrio, e 400 casos foram selecionados para compor a amostra. A coorte é constituída de casos tratados no Serviço de Oncologia Ginecológica do HC FMRP-USP de janeiro de 1998 até dezembro de 2017.

As pacientes foram consideradas elegíveis se tivessem câncer endometrial sem evidência clínica ou radiológica de doença cervical ou extrauterina. Os critérios de exclusão foram: doença avançada no diagnóstico, tratamento cirúrgico prévio para câncer de endométrio, cirurgia não realizada por outro motivo (por exemplo, status de desempenho comprometido), quimioterapia ou radioterapia prévia para tratamento de câncer de endométrio.

Foram excluídos 107 casos pelos seguintes motivos: 43 foram diagnosticados em estágios avançados, 8 apresentaram outro tumor primário concomitante, 11 foram submetidos à cirurgia vaginal, 34 não foram submetidos ao estadiamento cirúrgico em nosso serviço e em 9 não houve registro adequado do seguimento. As 400 pacientes restantes com presumível câncer endometrial precoce foram incluídas neste estudo, e do total, 316 foram submetidos à laparotomia convencional e 84 à cirurgia laparoscópica.

\section{Técnica cirúrgica}

Durante o período analisado, a cirurgia minimamente invasiva e a cirurgia aberta tradicional foram utilizadas em nosso serviço, de acordo com a preferência do cirurgião. A técnica cirúrgica videolaparoscópica utilizada está descrita nas próximas linhas. A paciente foi colocada na posição de litotomia, com inserção do manipulador uterino. Foi realizada incisão 
infra umbilical da pele, com introdução de uma agulha de Verres e expansão da cavidade abdominal com CO2, e após, colocação de um trocater de $12 \mathrm{~mm}$. A cavidade abdominal foi explorada e dois trocateres adicionais $(5 \mathrm{~mm})$ foram então inseridos nos quadrantes inferiores direito e esquerdo, ocasionalmente com inserção de um trocater adicional ( $5 \mathrm{~mm}$ ou $12 \mathrm{~mm}$ ) na linha média acima da sínfise púbica. A paciente foi então colocada em posição de Trendelenburg e a pelve foi explorada. Qualquer líquido pélvico identificado foi coletado para citologia; caso contrário, foi realizada uma lavagem com solução salina e posterior coleta. As tubas uterinas foram ligadas em sua porção média, com utilização do eletrocautério bipolar. Os ligamentos redondos foram seccionados bilateralmente. Foi feita uma incisão no peritônio sobre o músculo psoas, imediatamente lateral ao ligamento infundibulopélvico, com identificação do ureter, e os ligamentos infundibulopélvicos foram seccionados bilateralmente. A artéria e veia uterina foram identificadas e seccionadas. A bexiga foi mobilizada inferiormente e o peritônio sobrejacente à interface entre o reto e a vagina posterior foi incisado, expondo o espaço retovaginal. O pneumoperitônio foi esvaziado e a paciente reposicionada para litotomia inferior, sem Trendelemburg. Em seguida, procedeu-se à abordagem vaginal para abrir o manguito, seccionar os ligamentos uterossacros e paramétrios, e sutura da cúpula vaginal. A linfadenectomia pélvica laparoscópica foi realizada com base em avaliação histológica prévia e avaliação intraoperatória da invasão endometrial.

As incisões medianas foram usadas preferencialmente para as laparotomias. Nos dois tipos de cirurgias, a decisão sobre quando realizar a linfadenectomia foi baseada em imagem e biópsia pré-operatória (histologia e grau) e achados intraoperatórios (estimativa de invasão miometrial). Como regra geral, mulheres com tumores endometrióides superficiais grau I / II não foram submetidas à linfadenectomia.

\section{Variáveis}

As variáveis coletadas foram: tipo de cirurgia, idade ao diagnóstico, índice de massa corporal (IMC), volume uterino ultrassonográfico, histologia do tumor, estágio FIGO do tumor, grau do tumor, níveis de CA 125 no pré-operatório, tratamento adjuvante (radioterapia, quimioterapia), duração da anestesia, realização de linfadenectomia (pélvica e/ou para-aórtica), complicações intraoperatórias, complicações pós-operatórias cirúrgicas, complicações pósoperatórias clínicas, eventos tromboembólicos, transfusão de sangue necessária, internação 
pós-operatória hospitalar, sobrevida livre de doença (SLD) em dez anos e sobrevida global (SG) em dez anos.

A SLD foi definida como o intervalo entre o momento da cirurgia primária e a recorrência de qualquer doença documentada. A SG foi definida como o intervalo entre a cirurgia primária e a morte por qualquer causa. Para os pacientes que ainda estavam vivos no momento da análise, SLD e SG foram censurados na data do acompanhamento.

Banco de dados e análise estatística

Os dados estão armazenados em um banco de dados através da plataforma segura na web REDCap.

Variáveis qualitativas estão expressas em frequências e porcentagens. Para estimar a proporção de risco associada (hazard ratio) a cada fator prognóstico utilizamos o modelo de riscos proporcionais de Cox quando possível (COX, 1972). Curvas de sobrevida para períodos específicos foram construídas segundo o método de Kaplan-Meier. A manipulação dos dados e análise estatística foram realizadas com o R Studio Versão 1.2.1335. 
Figura 3 - Fluxograma da casuística do estudo

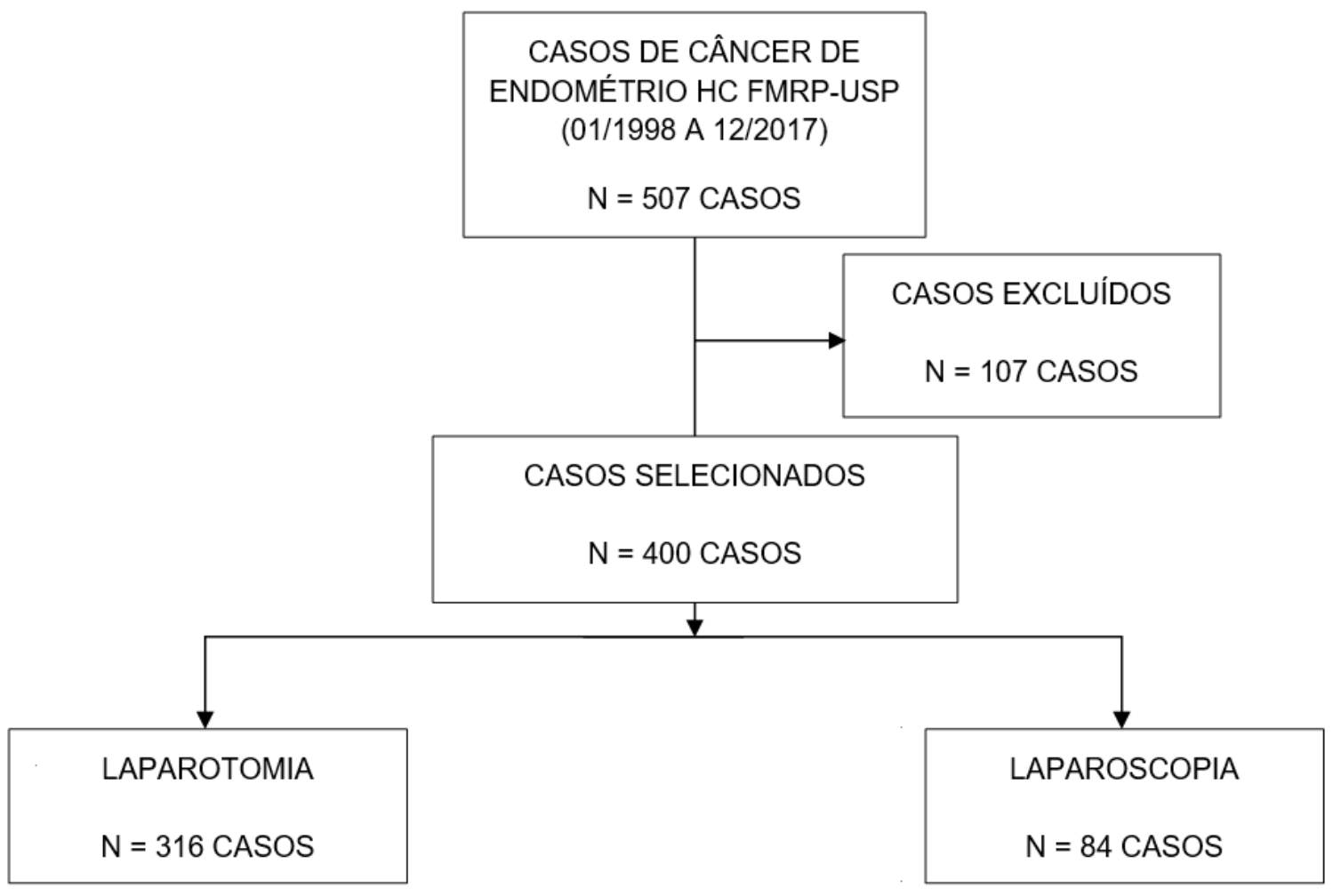

Fonte: Elaborado pelo autor. 


\section{ANEXO 2. Aprovação do Comitê de Ética.}

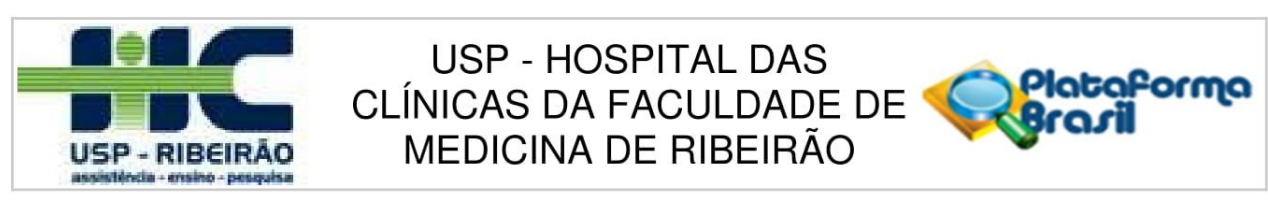

\section{PARECER CONSUBSTANCIADO DO CEP}

\section{DADOS DO PROJETO DE PESQUISA}

Título da Pesquisa: Cirurgia para o câncer de endométrio: comparação das abordagens laparotômica e laparoscópica

Pesquisador: Francisco José Candido dos Reis

Área Temática:

Versão: 1

CAAE: 81115817.5 .0000 .5440

Instituição Proponente: Hospital das Clínicas da Faculdade de Medicina de Ribeirão Preto da USP -

Patrocinador Principal: Financiamento Próprio

\section{DADOS DO PARECER}

\section{Número do Parecer: 2.462 .713}

\section{Apresentação do Projeto:}

O câncer de endométrio é o mais comum entre as mulheres nos Estados Unidos, representando $6 \%$ de todos os tumores malignos no sexo feminino. No Brasil, corresponde ao sexto mais frequente (COORDENAÇÃO, 2016). Está associado a fatores de risco bem definidos como obesidade, nuliparidade ou risco genético como a síndrome de Lynch (MORICE et al., 2016). Comparado a outros tumores, o prognóstico e a sobrevida das pacientes com câncer de endométrio é bom, com uma sobrevida em 10 anos de 77,6\% (Reino Unido) (PETO et al., 2000). Como o diagnóstico na maioria dos casos acontece em estádios iniciais, a cirurgia é curativa, assumindo papel crucial no tratamento desta patologia. Nas últimas duas décadas diversos aprimoramentos têm sido adicionados ao manejo das neoplasias ginecológicas. Estes aprimoramento referem-se praticamente a todas as etapas da atenção a paciente com neoplasias malignas, ou seja aumento na cobertura dos rastreamentos, melhorias nos fluxos hierarquizados de atendimento e notáveis avanços no conhecimento da biologia tumoral, com consequentes modificações nos protocolos de tratamento. Esta combinação de fatores têm permitido notáveis quedas na mortalidade por neoplasias.

Nos casos de cancer do endométrio o estadiamento e a abordagem terapêutica inicial requerem a realização de uma cirurgia. Nas últimas duas décadas ampliou-se de forma substancial o uso de técnicas minimamente invasivas como a videolaparoscopia e mais recentemente a cirurgia robótica para a cirurgia do câncer de endométrio. As técnicas minimamente invasivas são efetivas e

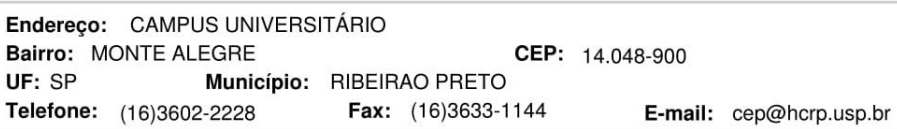




USP - HOSPITAL DAS
CLIINICAS DA FACULDADE DE R Platoforma
MEDICINA DE RIBEIRÃO

Continuação do Parecer: 2.462 .713

reduzem as complicações relacionadas à abordagem cirúrgica. Diversos estudos apontam vantagens da técnica laparoscópica sobre a laparotomia convencional. As principais vantagens em curto prazo referem-se a redução de dor e melhor qualidade de vida (JANDA et al., 2010; KORNBLITH et al., 2009; OBERMAIR et al., 2005). Alguns riscos também podem estar associados às técnicas laparoscópicas como o risco de lesão intra-operatória em pacientes obesas ou o implante de tumor em sítios de punção. Poucos estudos investigam os efeitos de longo prazo do uso de técnicas minimamente invasivas. As evidências apontam no sentido de equivalência em termos de sobrevida em longo prazo entre a laparotomia e a laparoscopia (GALAAL et al., 2012; JANDA et al., 2017), com benefícios de curto prazo para a técnica minimamente invasiva. Estudar coortes de seguimento onde ambos os métodos foram aplicados concomitantemente na prática real é fundamental vara se estimar os efeitos populacionais da implantação das técnicas minimamente invasivas fora de estudos clínicos randomizados.

\section{Objetivo da Pesquisa:}

Hipótese:

A laparoscopia não é inferior à laparotomia para a abordagem do cancer de endométrio

Objetivo Primário:

Comparar as técnicas laparotômica e laparoscópicas em uma coorte de tumores de endométrio.

Objetivo Secundário:

Comparar os parâmetros intra-operatórios das técnicas laparoscópicas e laparotômicas para o estadiamento cirúrgico do câncer de endométrio. Comparar resultados de curto prazo das técnicas laparoscópicas e laparotômicas para o estadiamento cirúrgico do câncer de endométrio. Avaliar o risco de recorrência de médio e longo prazo em mulheres submetidas a laparoscopia e laparotomia para o câncer de endométrio. Avaliar a sobrevida em mulheres submetidas a laparoscopia e laparotomia para o câncer de endométrio.

\section{Avaliação dos Riscos e Benefícios:}

Esta pesquisa não acarretará exposição dos pacientes a quaisquer riscos, pois se trata de estudo envolvendo coleta de informações clínicas existentes em prontuário das pacientes do Hospital das Clínicas de Ribeirão Preto. Os pesquisadores comprometeram-se a manter o sigilo das pacientes durante a pesquisa. Como não haverá intervenção direta, também não são esperados benefícios diretos para as pacientes.

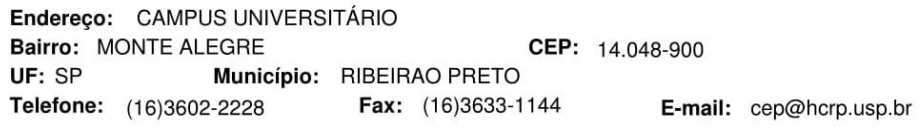




USP - HOSPITAL DAS
CLÍNICAS DA FACULDADE DE Q
MEDICINA DE RIBEIRÃO

Continuação do Parecer: 2.462 .713

\section{Comentários e Considerações sobre a Pesquisa:}

Metodologia Proposta:

Trata-se de um estudo observacional populacional tipo coorte. Os sujeitos do estudo são mulheres com confirmação histológica de tumores de endométrio. As participantes são originárias do serviço de oncologia ginecológica do Hospital das Clínicas da Faculdade de Medicina de Ribeirão Preto. A coorte é constituída de aproximadamente 400 casos tratados no Serviço de Oncologia Ginecológica do Hospital das Clínicas da Faculdade de Medicina de Ribeirão Preto de 1998 até 2017. Os dados demográficos, ano de diagnóstico, dados relativos à localização do tumor primário, tipo histológico do tumor, estádio, tipo de tratamento e tempo de sobrevida serão obtidos de registros médicos. Pesquisa retrospectiva que poderá auxiliar na decisão da melhor abordagem cirúrgica em pacientes com câncer de endométrio.

Considerações sobre os Termos de apresentação obrigatória:

Todos os termos estão de acordo com as normas éticas em pesquisa.

Recomendações:

Não há.

Conclusões ou Pendências e Lista de Inadequações:

Diante do exposto e à luz da Resolução CNS 466/2012, o projeto de pesquisa, assim como a dispensa do Termo de Consentimento Livre e Esclarecido, podem ser enquadrados na categoria APROVADO.

\section{Considerações Finais a critério do CEP:}

Projeto Aprovado: Tendo em vista a legislação vigente, devem ser encaminhados ao CEP, relatórios parciais anuais referentes ao andamento da pesquisa e relatório final ao término do trabalho. Qualquer modificação do projeto original deve ser apresentada a este CEP em nova versão, de forma objetiva e com justificativas, para nova apreciação.

Este parecer foi elaborado baseado nos documentos abaixo relacionados:

\begin{tabular}{|l|l|c|l|c|}
\hline \multicolumn{1}{|c|}{ Tipo Documento } & \multicolumn{1}{|c|}{ Arquivo } & Postagem & Autor & Situação \\
\hline $\begin{array}{l}\text { Informações Básica } \\
\text { do Projeto }\end{array}$ & PB_INFORMAÇÕES_BÁSICAS_DO_P & $\begin{array}{c}14 / 12 / 2017 \\
08: 37: 22\end{array}$ & & Aceito \\
\hline Outros & ROJETO 1020102.pdf & $\begin{array}{c}14 / 12 / 2017 \\
08: 36: 04\end{array}$ & $\begin{array}{l}\text { Francisco José } \\
\text { Candido dos Reis }\end{array}$ & Aceito \\
\hline TCLE / Termos de & Aprovacao_DGO.pdf & $\begin{array}{c}14 / 12 / 2017 \\
08: 35: 25\end{array}$ & $\begin{array}{l}\text { Francisco José } \\
\text { Candido dos Reis }\end{array}$ & Aceito \\
Assentimento / & & \multicolumn{2}{|c|}{} \\
\hline
\end{tabular}

Endereço: CAMPUS UNIVERSITÁRIO

Bairro: MONTE ALEGRE

UF: SP Município: RIBEIRAO PRETO

Telefone: (16)3602-2228 Fax: (16)3633-1144 E-mail: cep@hcrp.usp.br 


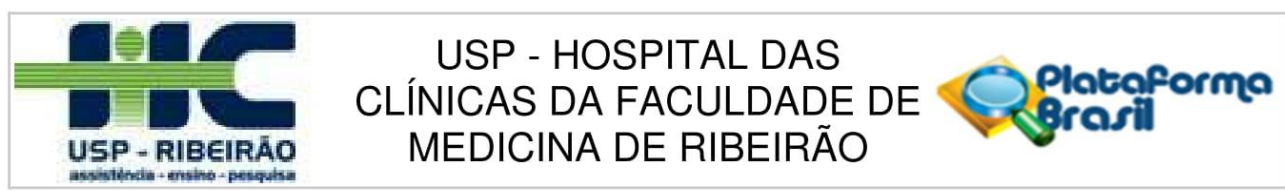

Continuação do Parecer: 2.462 .713

\begin{tabular}{|c|c|c|c|c|}
\hline \begin{tabular}{|l|} 
Justificativa de \\
Ausência \\
\end{tabular} & Dispensa_TCLE.pdf & $\begin{array}{c}14 / 12 / 2017 \\
08: 35: 25 \\
\end{array}$ & \begin{tabular}{|l|} 
Francisco José \\
Candido dos Reis \\
\end{tabular} & Aceito \\
\hline $\begin{array}{l}\text { Projeto Detalhado / } \\
\text { Brochura } \\
\text { Investigador }\end{array}$ & 21071020_Projeto_Licerio.pdf & $\begin{array}{c}14 / 12 / 2017 \\
08: 33: 12\end{array}$ & $\begin{array}{l}\text { Francisco José } \\
\text { Candido dos Reis }\end{array}$ & Aceito \\
\hline Orçamento & Aprovacao_UPC.PDF & $\begin{array}{c}14 / 12 / 2017 \\
08: 31: 02 \\
\end{array}$ & $\begin{array}{l}\text { Francisco José } \\
\text { Candido dos Reis }\end{array}$ & Aceito \\
\hline Folha de Rosto & Folha_de_rosto_assinada.pdf & $\begin{array}{c}14 / 12 / 2017 \\
08: 29: 41 \\
\end{array}$ & $\begin{array}{l}\text { Francisco José } \\
\text { Candido dos Reis }\end{array}$ & Aceito \\
\hline
\end{tabular}

Situação do Parecer:

Aprovado

Necessita Apreciação da CONEP:

Não

RIBEIRAO PRETO, 09 de Janeiro de 2018

Assinado por:

MARCIA GUIMARÃES VILLANOVA

(Coordenador)

Endereço: CAMPUS UNIVERSITÁRIO

Bairro: MONTE ALEGRE

CEP: $\quad 14.048-900$

UF: SP Município: RIBEIRAO PRETO

Telefone: (16)3602-2228 Fax: (16)3633-1144 E-mail: cep@hcrp.usp.br

Página 04 de 04 
5 Referências 
ALLEN, N. E. et al. Menopausal Hormone Therapy and Risk of Endometrial Carcinoma Among Postmenopausal Women in the European Prospective Investigation into Cancer and Nutrition. American Journal of Epidemiology, v. 172, n. 12, p. 1394-1403, 15 dez. 2010.

AMANT, F. et al. Endometrial cancer. Lancet (London, England), v. 366, n. 9484, p. 491505, 6 ago. 2005.

BELL, D. W.; ELLENSON, L. H. Molecular Genetics of Endometrial Carcinoma. p. 29, 2018.

BERGSTRÖM, A. et al. Overweight as an avoidable cause of cancer in Europe. Int J Cancer, v. 91, n. 3, p. 421-30, fev. 2001.

BRAUN, M. M. Diagnosis and Management of Endometrial Cancer. Endometrial Cancer, v. 93 , n. 6, p. 7, 2016.

BURKE, W. M. et al. Endometrial cancer: A review and current management strategies: Part I. Gynecologic Oncology, v. 134, n. 2, p. 385-392, ago. 2014a.

BURKE, W. M. et al. Endometrial cancer: A review and current management strategies: Part II. Gynecologic Oncology, v. 134, n. 2, p. 393-402, ago. 2014b.

CARDENAS-GOICOECHEA, J. et al. Survival analysis of robotic versus traditional laparoscopic surgical staging for endometrial cancer. American Journal of Obstetrics and Gynecology, v. 210, n. 2, p. 160.e1-160.e11, fev. 2014.

CHLEBOWSKI, R. T. et al. Aromatase inhibitors, tamoxifen, and endometrial cancer in breast cancer survivors: Aromatase Inhibitors and Endometrial CA. Cancer, v. 121, n. 13, p. 2147-2155, 1 jul. 2015.

Clinical Management Guidelines for Obstetrician-Gynecologists. Number 42, April 2003:

Breast Cancer Screening. Obstetrics \& Gynecology, v. 101, n. 4, p. 821-832, abr. 2003.

COORDENAÇÃO, I. N. DE C. J. A. G. DA SILVA. Estimate/2016 - Cancer Incidence in Brazil. [s.l: s.n.].

COX, D. R. Regression Models and Life-Tables. Journal of the Royal Statistical Society. Series B (Methodological), v. 34, n. 2, p. 187-220, 1972.

CREUTZBERG, C. L. et al. Surgery and postoperative radiotherapy versus surgery alone for patients with stage-1 endometrial carcinoma: multicentre randomised trial. THE LANCET, v. 355 , p. $8,2000$.

CREUTZBERG, C. L. et al. Fifteen-Year Radiotherapy Outcomes of the Randomized PORTEC-1 Trial for Endometrial Carcinoma. International Journal of Radiation Oncology*Biology*Physics, v. 81, n. 4, p. e631-e638, nov. 2011.

CRISPENS, M. Endometrial and Ovarian Cancer in Lynch Syndrome. Clinics in Colon and Rectal Surgery, v. 25, n. 02, p. 097-102, jun. 2012.

ELTABBAKH, G. H. et al. Hysterectomy for Obese Women with Endometrial Cancer: Laparoscopy or Laparotomy? Gynecologic Oncology, v. 78, n. 3, p. 329-335, set. 2000. 
ELTABBAKH, G. H.; SHAMONKI, J.; MOUNT, S. L. Surgical stage, final grade, and survival of women with endometrial carcinoma whose preoperative endometrial biopsy shows well-differentiated tumors. Gynecologic Oncology, v. 99, n. 2, p. 309-312, nov. 2005.

EVERETT, E. et al. The effect of body mass index on clinical/pathologic features, surgical morbidity, and outcome in patients with endometrial cancer. Gynecol Oncol, v. 90, n. 1, p. 150-7, jul. 2003.

FELIX, A. S. et al. High cardiovascular disease mortality after endometrial cancer diagnosis: Results from the Surveillance, Epidemiology, and End Results (SEER) Database.

International Journal of Cancer, v. 140, n. 3, p. 555-564, 2017.

FERLAY, J. et al. Cancer incidence and mortality worldwide: sources, methods and major patterns in GLOBOCAN 2012. Int J Cancer, v. 136, n. 5, p. E359-86, mar. 2015.

FOLEY, K.; LEE, R. B. Surgical complications of obese patients with endometrial carcinoma. Gynecol Oncol, v. 39, n. 2, p. 171-4, nov. 1990.

GALAAL, K. et al. Laparoscopy versus laparotomy for the management of early stage endometrial cancer. The Cochrane Database of Systematic Reviews, n. 9, p. CD006655, 12 set. 2012a.

GALAAL, K. et al. Adjuvant chemotherapy for advanced endometrial cancer. Cochrane Database of Systematic Reviews, 15 maio 2014.

GREDMARK, T. et al. Histopathological findings in women with postmenopausal bleeding. BJOG: An International Journal of Obstetrics and Gynaecology, v. 102, n. 2, p. 133-136, fev. 1995.

HARRIS, H. R.; TERRY, K. L. Polycystic ovary syndrome and risk of endometrial, ovarian, and breast cancer: a systematic review. Fertility Research and Practice, v. 2, n. 1, p. 14, dez. 2016.

INOUE, S. et al. Improvement in 5-Year Relative Survival in Cancer of the Corpus Uteri From 1993-2000 to 2001-2006 in Japan. Journal of Epidemiology, v. 28, n. 2, p. 75-80, 5 fev. 2018.

JANDA, M. et al. Quality of life after total laparoscopic hysterectomy versus total abdominal hysterectomy for stage I endometrial cancer (LACE): a randomised trial. The Lancet.

Oncology, v. 11, n. 8, p. 772-780, ago. 2010.

JANDA, M. et al. Effect of Total Laparoscopic Hysterectomy vs Total Abdominal Hysterectomy on Disease-Free Survival Among Women With Stage I Endometrial Cancer: A Randomized Clinical Trial. JAMA, v. 317, n. 12, p. 1224-1233, 28 mar. 2017.

JOHNSON, L. et al. Clinical comparison of robotic, laparoscopic, and open hysterectomy procedures for endometrial cancer patients. Journal of Robotic Surgery, v. 11, n. 3, p. 291297, set. 2017.

KEYS, H. M. et al. A phase III trial of surgery with or without adjunctive external pelvic radiation therapy in intermediate risk endometrial adenocarcinoma: a Gynecologic Oncology Group study. Gynecologic Oncology, v. 92, n. 3, p. 744-751, mar. 2004. 
KIM, H.-S.; LEE, S.; KIM, J. H. Real-world Evidence versus Randomized Controlled Trial: Clinical Research Based on Electronic Medical Records. Journal of Korean Medical Science, v. 33, n. 34, 26 jun. 2018.

KOH, W.-J.; DORIGO, O.; MUTCH, D. NCCN Guidelines Index Table of Contents Discussion. p. 105, 2017.

KORNBLITH, A. B. et al. Quality of life of patients with endometrial cancer undergoing laparoscopic international federation of gynecology and obstetrics staging compared with laparotomy: a Gynecologic Oncology Group study. Journal of Clinical Oncology: Official Journal of the American Society of Clinical Oncology, v. 27, n. 32, p. 5337-5342, 10 nov. 2009.

LEE, Y. C.; LHEUREUX, S.; OZA, A. M. Treatment strategies for endometrial cancer: current practice and perspective. Current Opinion in Obstetrics and Gynecology, v. 29, n. 1, p. 47-58, fev. 2017.

LESTER-COLL, N. H. et al. Adjuvant Therapy Use and Survival in Stage II Endometrial Cancer. International Journal of Gynecologic Cancer, v. 27, n. 9, p. 1904-1911, nov. 2017.

LYNCH, H. T. et al. Milestones of Lynch syndrome: 1895-2015. Nature Reviews Cancer, v. 15, n. 3, p. 181-194, mar. 2015.

MOORE, K.; BREWER, M. A. Endometrial Cancer: Is This a New Disease? ENDOMETRIAL CANCER, p. 8, [s.d.].

MORICE, P. et al. Endometrial cancer. Lancet (London, England), v. 387, n. 10023, p. 1094-1108, 12 mar. 2016.

MOURITS, M. J. E. et al. Safety of laparoscopy versus laparotomy in early-stage endometrial cancer: a randomised trial. The Lancet. Oncology, v. 11, n. 8, p. 763-771, ago. 2010.

MUTCH, D. G. The new FIGO staging system for cancers of the vulva, cervix, endometrium and sarcomas. Gynecologic Oncology, v. 115, n. 3, p. 325-328, dez. 2009.

NOUT, R. et al. Vaginal brachytherapy versus pelvic external beam radiotherapy for patients with endometrial cancer of high-intermediate risk (PORTEC-2): an open-label, noninferiority, randomised trial. The Lancet, v. 375, n. 9717, p. 816-823, mar. 2010.

OBERMAIR, A. et al. Total laparoscopic hysterectomy versus total abdominal hysterectomy for obese women with endometrial cancer. International Journal of Gynecological Cancer: Official Journal of the International Gynecological Cancer Society, v. 15, n. 2, p. 319324, abr. 2005.

PALOMBA, S. et al. Updating of a recent meta-analysis of randomized controlled trials to assess the safety and the efficacy of the laparoscopic surgery for treating early stage endometrial cancer. Gynecologic Oncology, v. 114, n. 1, p. 135-136, jul. 2009.

PETO, R. et al. UK and USA breast cancer deaths down 25\% in year 2000 at ages 20-69 years. Lancet, v. 355, n. 9217, p. 1822, maio 2000. 
REIJNEN, C. et al. Improved preoperative risk stratification with CA-125 in low-grade endometrial cancer: a multicenter prospective cohort study. Journal of Gynecologic Oncology, v. 30, n. 5, p. e70, 2019.

ROSE, P. G. Endometrial Carcinoma. New England Journal of Medicine, v. 335, n. 9, p. 640-649, 29 ago. 1996.

SANTOS, M. DE O. Estimativa 2018: Incidência de Câncer no Brasil. Revista Brasileira de Cancerologia, v. 64, n. 1, p. 119-120, 30 mar. 2018.

SCHRAMM, A. et al. Value of endometrial thickness assessed by transvaginal ultrasound for the prediction of endometrial cancer in patients with postmenopausal bleeding. Archives of Gynecology and Obstetrics, v. 296, n. 2, p. 319-326, ago. 2017.

SÉNÉCHAL, C. et al. Les facteurs de risque génétiques et environnementaux des cancers de l'endomètre. Bulletin du Cancer, v. 102, n. 3, p. 256-269, mar. 2015.

SIEGEL, R. L.; MILLER, K. D.; JEMAL, A. Cancer statistics, 2019. CA: a cancer journal for clinicians, v. 69, n. 1, p. 7-34, jan. 2019.

SOBCZUK, K.; SOBCZUK, A. New classification system of endometrial hyperplasia WHO 2014 and its clinical implications. Menopausal Review, v. 3, p. 107-111, 2017.

SOROSKY, J. I. Endometrial Cancer: Obstetrics \& Gynecology, v. 120, n. 2, Part 1, p. 383-397, ago. 2012.

TOZZI, R. et al. Laparoscopy versus laparotomy in endometrial cancer: first analysis of survival of a randomized prospective study. J Minim Invasive Gynecol, v. 12, n. 2, p. 130-6, abr. 2005.

WALKER, J. L. et al. Laparoscopy Compared With Laparotomy for Comprehensive Surgical Staging of Uterine Cancer: Gynecologic Oncology Group Study LAP2. Journal of Clinical Oncology, v. 27, n. 32, p. 5331-5336, 10 nov. 2009.

WALKER, J. L. et al. Recurrence and Survival After Random Assignment to Laparoscopy Versus Laparotomy for Comprehensive Surgical Staging of Uterine Cancer: Gynecologic Oncology Group LAP2 Study. Journal of Clinical Oncology, v. 30, n. 7, p. 695-700, mar. 2012.

WARD, K. K. et al. Cardiovascular disease is the leading cause of death among endometrial cancer patients. Gynecologic Oncology, v. 126, n. 2, p. 176-179, ago. 2012.

WHITE, I. R.; ROYSTON, P.; WOOD, A. M. Multiple imputation using chained equations: Issues and guidance for practice. Statistics in Medicine, v. 30, n. 4, p. 377-399, 20 fev. 2011.

WRIGHT, J. D. et al. Use and Benefits of Laparoscopic Hysterectomy for Stage I Endometrial Cancer Among Medicare Beneficiaries. Journal of Oncology Practice, v. 8, n. 5, p. e89-e99, 19 jun. 2012.

ZULLO, F. et al. A prospective randomized comparison between laparoscopic and laparotomic approaches in women with early stage endometrial cancer: a focus on the quality 
of life. American Journal of Obstetrics and Gynecology, v. 193, n. 4, p. 1344-1352, out. 2005.

ZULLO, F.; FALBO, A.; PALOMBA, S. Safety of laparoscopy vs laparotomy in the surgical staging of endometrial cancer: a systematic review and metaanalysis of randomized controlled trials. American Journal of Obstetrics and Gynecology, v. 207, n. 2, p. 94-100, ago. 2012. 